\title{
Broadband Transmission and Statistical Performance Properties of Overhead High-Voltage Transmission Networks
}

\author{
Athanasios G. Lazaropoulos \\ School of Electrical and Computer Engineering, National Technical University of Athens, 15780 Zografou, Greece \\ Correspondence should be addressed to Athanasios G. Lazaropoulos, aglazaropoulos@gmail.com
}

Received 19 October 2011; Revised 17 January 2012; Accepted 5 February 2012

Academic Editor: Leszek Szczecinski

Copyright (C) 2012 Athanasios G. Lazaropoulos. This is an open access article distributed under the Creative Commons Attribution License, which permits unrestricted use, distribution, and reproduction in any medium, provided the original work is properly cited.

This paper considers broadband signal transmission and statistical performance properties of high-voltage/broadband over power lines (HV/BPL) channels associated with overhead power transmission. The overhead HV/BPL transmission channel is investigated with regard to its spectral behavior, its end-to-end signal attenuation, and its statistical performance metrics. It is found that the above features depend critically on the frequency, the overhead HV power grid type $(150 \mathrm{kV}, 275 \mathrm{kV}$, or $400 \mathrm{kV}$ and single- or double-circuit), the coupling scheme applied, the physical properties of the cables used, the MTL configuration, and the type of branches existing along the end-to-end BPL signal propagation. The contribution of this paper is threefold. First, the significant broadband transmission potential of overhead HV lines is revealed. The results demonstrate that, regardless of overhead HV power grid type, the overhead HV grid is a potentially excellent communications medium, offering low-loss characteristics, flat-fading features, and low multipath dispersion over a $25 \mathrm{~km}$ repeater span well beyond $100 \mathrm{MHz}$. Second, regarding the statistical properties of various overhead HV/BPL transmission channels, two fundamental correlations of several wireline systems, for example, coaxial cables and xDSL, are also validated in the case of overhead HV/BPL transmission channels, namely, (i) end-toend channel attenuation in relation with root-mean-square delay spread (RMS-DS) and (ii) coherence bandwidth (CB) in relation with RMS-DS. Third, fitting the numerical results and other field trial measurements, two regression distributions suitable for each fundamental correlation are proposed.

\section{Introduction}

The ubiquitous presence of the low-voltage (LV), mediumvoltage (MV), and high-voltage (HV) power grids is the key to developing an advanced smart grid (SG) power network, offering a plethora of potential SG applications, such as ubiquitous grid surveillance at small cost, continuous monitoring, real-time adjustment of sensitive loads, and optimal response to power demand during critical circumstances [ 1 , 2]. Moreover, the deployment of broadband over power lines (BPL) networks through the entire grid forms a potentially convenient and inexpensive communication medium for delivering broadband last mile access in remote and/or underdeveloped areas [3].

With the goal of providing operational telephone services and data communications across large geographical distances, the first power line communications (PLC) efforts were put in place by power utilities over HV power grid in the early 1920s $[4,5]$. Due to the upcoming SG adaption both in transmission and distribution power networks, the availability of a reliable communication network on the $\mathrm{HV}$ power grid side is important for the support of these significant changes [6-9]. Since overhead HV power lines are generally the lowest-cost method of transmission for large quantities of electric power, utilities employ primarily overhead HV transmission power grid for new urban, suburban, and rural installations [9-13].

The modeling problem for overhead HV/BPL channels, which consists of investigating the characteristics of power network as a communication medium, is a difficult challenge because reliable high-speed communication is sought over a medium designed for electrical energy delivery rather than 
for data transmission. When considered as a transmission medium for communications signals, the overhead HV power grid is subjected to attenuation, multipath due to various reflections, noise, and electromagnetic interference (EMI) [14-21]. Each of the aforementioned adverse factors affects critically the overall performance and the design of BPL systems [22, 23].

Due to the evolution of broadband SG requirements, the development of accurate channel models at high frequencies along the $\mathrm{HV}$ transmission power lines is imperative. As usually done in BPL transmission, a hybrid model is employed to examine the behavior of BPL transmission channels installed on BPL multiconductor transmission line (MTL) structures [14, 15, 24-31]. This hybrid model follows (i) a bottomup approach consisting of an appropriate combination of similarity transformations and MTL theory to determine the propagation constant and the characteristic impedance of the modes supported [24-37] and (ii) a top-down approach based on cascaded matrices of two-port network modulesthe $T$-matrix (TM) method-to determine the end-to-end attenuation of BPL channel connections $[10,15,16,22$, $24,26,28,30,34,37,38]$. The influence of factors, such as the overhead HV power grid type $(150 \mathrm{kV}, 275 \mathrm{kV}$, or $400 \mathrm{kV}$ and single- or double-circuit), the coupling scheme applied, the physical properties of the cables used, the MTL configuration, the end-to-end distance, and the number and the electrical length encountered along the end-toend HV/BPL signal propagation, is investigated based on numerical results concerning simulated overhead HV/BPL topologies.

Taking into account the overhead HV/BPL simulation results, the statistical properties of HV/BPL channels can be studied. The common nature among HV/BPL, MV/BPL, and LV/BPL systems is reflected on important statistical performance metrics, such as the average end-to-end channel attenuation, the root-mean-square delay spread (RMSDS), and the coherence bandwidth (CB). Specifically, it is confirmed that (i) average end-to-end channel attenuation and RMS-DS in overhead HV/BPL channels are positively correlated lognormal random variables being in agreement with relevant sets of measurements and relevant distributions of recently proposed statistical BPL channel models [39-47]. On the basis of the confirmed correlation, a new approximation- $\mathrm{UNI}^{1}$ approach — suitable for the design of BPL transmission and distribution power grids is proposed; (ii) $\mathrm{CB}$ and RMS-DS correlation behavior in overhead HV/BPL can be described by hyperbolic functions. By fitting the simulation results, a new approximation- $-\mathrm{UNI}^{2}$ approach - is proposed appropriate for overhead HV/BPL systems which is compared with recently proposed statistical distributions for various BPL systems and for different $\mathrm{CB}$ correlation levels [44-48]. The commonality of the above mentioned correlations in several wireline communications channels is also confirmed in overhead HV/BPL transmission case.

The rest of this paper is organized as follows. In Section 2, the modal and the coupling scheme spectral behaviors of overhead HV/BPL broadband propagation are discussed along with the necessary assumptions concerning BPL trans- mission. Section 3 provides a description of the statistical performance metrics considered in the rest of the paper. In Section 4, numerical results are provided, aiming at marking out how the various features of the overhead HV transmission power grids influence BPL transmission and statistical performance metrics. On the basis of the confirmed fundamental correlations between channel attenuation/RMSDS and CB/RMS-DS, new regression approximations are proposed. Section 5 concludes the paper.

\section{The Physical BPL Layer}

The overhead HV power grid differs considerably from transmission via twisted-pair, coaxial, or fiber-optic cables due to the significant differences of the network structure and the physical properties of the power cables used $[1,10,16,19$, $24,26,27,38]$.

Overhead HV transmission grid accommodates the transfer of hundreds or thousands MW of electric power in long distances (hundreds or thousands $\mathrm{km}$ ) from the generators to HV/MV substations. These substations define the boundaries between transmission and distribution power grid sides. Overhead HV power transmission systems are mainly classified in the electrical power industry by (i) their voltage levels (from $150 \mathrm{kV}$ up to $1 \mathrm{MV}$ ) and (ii) their number of MTL circuits per tower (mainly, either single- or double-circuit). In the case of single-circuit threephase overhead HV systems, each tower supports threephase conductors whereas in the case of double-circuit threephase overhead HV systems, each tower supports six-phase conductors, and they are classified by (iii) the number of neutral conductors per tower [52].

A typical case of $150 \mathrm{kV}$ single-circuit overhead HV transmission line is depicted in Figure 1(a). Three parallel phase conductors spaced by $\Delta_{p}^{150 \mathrm{kV}}$ in the range from $6.60 \mathrm{~m}$ to $8.95 \mathrm{~m}$ are suspended at heights $h_{p}^{150 \mathrm{kV}}$ ranging from $19 \mathrm{~m}$ to $19.95 \mathrm{~m}$ above lossy ground-conductors 1,2 , and 3. Moreover, two parallel neutral conductors spaced by $\Delta_{n}^{150 \mathrm{kV}}$ in the range from $9.30 \mathrm{~m}$ to $12.10 \mathrm{~m}$ hang at heights $h_{n}^{150 \mathrm{kV}}$ ranging from $23.75 \mathrm{~m}$ to $24.7 \mathrm{~m}$-conductors 4 and 5 . This three-phase five-conductor $\left(n^{150 \mathrm{kV}}=5\right)$ overhead HV distribution line configuration is considered in the present work consisting of ACSR GROSBEK $3 \times 374.77 \mathrm{~mm}^{2}+2 \times$ $322.26 \mathrm{~mm}^{2}$ conductors [13, 49-51].

A typical case of overhead $275 \mathrm{kV}$ double-circuit overhead HV transmission line is illustrated in Figure 1(b). Two sets of three-phase conductors-conductors 1, 2, 3 and conductors $4,5,6$ - are spaced by $\Delta_{p 1}^{275 \mathrm{kV}}$ in the range from $8.84 \mathrm{~m}$ to $13.1 \mathrm{~m}$. The three-phase conductors of each set with radii $r_{p}^{275 \mathrm{kV}}=12.08 \mathrm{~mm}$ are suspended one above the other spaced by $\Delta_{p 2}^{275 \mathrm{kV}}$ ranging from $3.66 \mathrm{~m}$ to $5.18 \mathrm{~m}$ and located at heights $h_{p}^{275 \mathrm{kV}}$ in the range from $18.9 \mathrm{~m}$ to $20.48 \mathrm{~m}$ above ground for the lowest conductor. Apart from the six-phase conductors of two sets, the upper conductorconductor 7-is the neutral conductor with radii $r_{n}^{275 \mathrm{kV}}=$ $7.11 \mathrm{~mm}$, spaced by $\Delta_{n}^{275 \mathrm{kV}}$ ranging from $2.4 \mathrm{~m}$ to $3.08 \mathrm{~m}$ from the highest phase conductor-conductor 3 or 6 , and, consequently, hangs at heights $h_{n}^{275 \mathrm{kV}}$ in the range from 


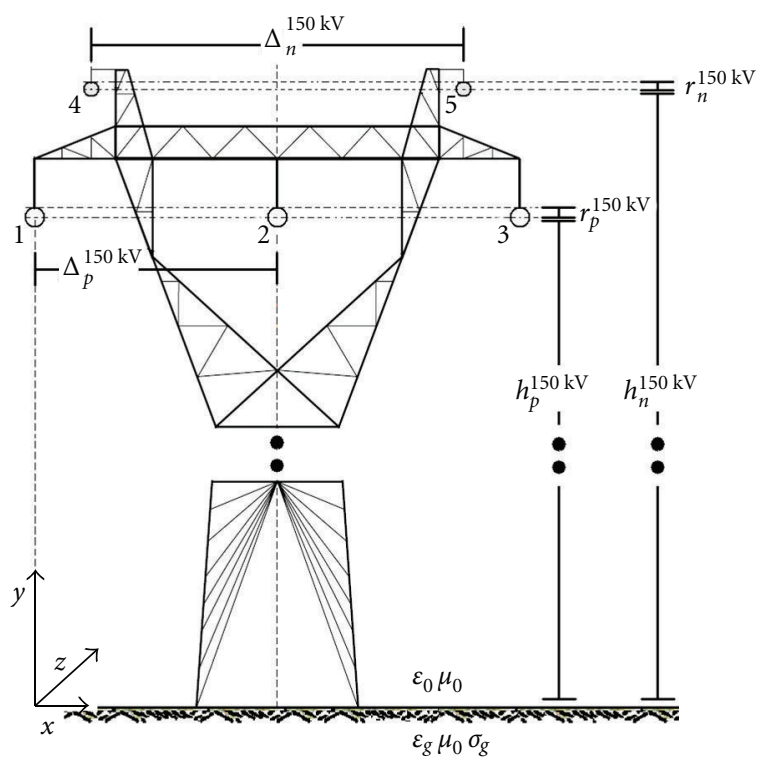

(a)

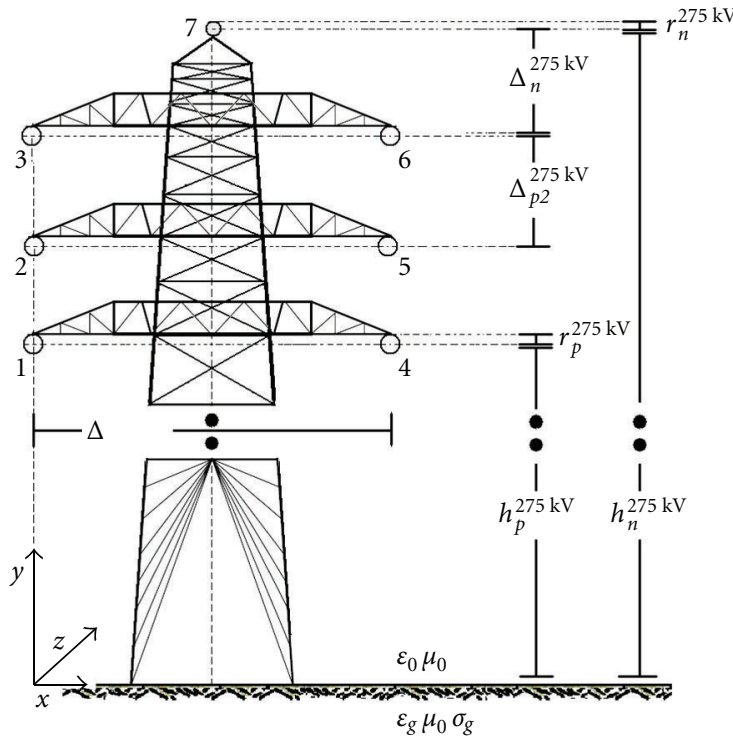

(b)

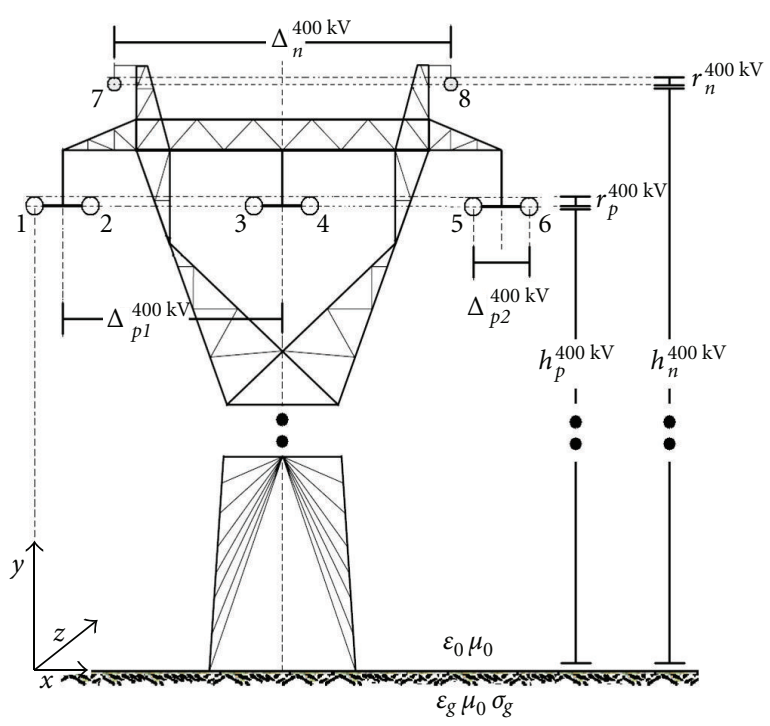

(c)

Figure 1: Typical overhead HV multiconductor structures [13, 24, 49-51]. (a) $150 \mathrm{kV}$ single-circuit. (b) $275 \mathrm{kV}$ double-circuit. (c) $400 \mathrm{kV}$ double-circuit.

$28.62 \mathrm{~m}$ to $33.92 \mathrm{~m}$ above ground. This double-circuit sevenconductor $\left(n^{275 \mathrm{kV}}=7\right)$ overhead HV distribution line configuration is considered in the present work consisting of ACSR conductors [13, 49-51, 53, 54].

Overhead $400 \mathrm{kV}$ double-circuit overhead HV transmission phase lines with radii $r_{p}^{400 \mathrm{kV}}=15.3 \mathrm{~mm}$ hang at typical heights $h_{p}^{400 \mathrm{kV}}$ equal to $20 \mathrm{~m}$ above ground -conductors 1 , $2,3,4,5$, and 6 . These six-phase conductors are divided into three bundles; the phase conductors of each bundle are connected by nonconducting spacers and are separated by $\Delta_{p 1}^{400 \mathrm{kV}}$ equal to $400 \mathrm{~mm}$, whereas bundles are spaced by
$\Delta_{p 2}^{400 \mathrm{kV}}$ equal to $10 \mathrm{~m}$. Moreover, two parallel neutral conductors with radii $r_{n}^{400 \mathrm{kV}}=9 \mathrm{~mm}$ spaced by $\Delta_{n}^{400 \mathrm{kV}}$ equal to $12 \mathrm{~m}$ hang at heights $h_{n}^{400 \mathrm{kV}}$ equal to $23.7 \mathrm{~m}$-conductors 7 and 8. This double-circuit eight-conductor $\left(n^{400 \mathrm{kV}}=8\right)$ overhead HV distribution line configuration is considered in the present work consisting of ACSR conductors-see Figure 1(c) [13, 49-52].

The ground is considered as the reference conductor. The conductivity of the ground is assumed $\sigma_{g}=5 \mathrm{mS} / \mathrm{m}$ and its relative permittivity $\varepsilon_{r g}=13$, which is a realistic scenario $[1,14,24,26,27,38]$. The impact of imperfect 
ground on signal propagation via overhead power lines was analyzed in $[14,26,27,38,55-58]$. This formulation has the advantage that, contrary to other available models for overhead power lines [59-62], it is suitable for transmission at high frequencies above lossy ground and for broadband applications of overhead HV/BPL, MV/BPL, and LV/BPL systems.

Through a matrix approach, the standard TL analysis can be extended to the MTL case which involves more than two conductors. Compared to a two-conductor line supporting one forward- and one backward-traveling wave, an MTL structure with $n+1$ conductors parallel to the $z$ axis as depicted in Figures 1(a), 1(b), and 1(c) may support $n$ pairs of forward- and backward-traveling waves with corresponding propagation constants. These waves may be described by a coupled set of $2 n$ first-order partial differential equations relating the line voltages $V_{i}(z, t), i=1, \ldots, n$ to the line currents $I_{i}(z, t), i=1, \ldots, n$. Each pair of forward- and backward-traveling waves is referred to as a mode [24, 26, 32, 33].

Consequently, in the case of overhead HV transmission lines involving $n$ conductors over lossy plane ground, $n$ modes may be supported, namely, as follows $[1,2,9,12,13$, 24, 26-29, 32-35, 38, 49, 55-58, 63, 64].

(i) Common mode (CM, $i=1$ ) of overhead HV/BPL transmission which propagates via the $n$ conductors and returns via the ground. $\gamma_{\mathrm{CM}}$ constitutes the $\mathrm{CM}$ propagation constant.

(ii) Differential modes $\left(\mathrm{DM}_{i-1}, i=2, \ldots, n\right)$ of overhead HV/BPL transmission which propagate and return via the $n$ conductors. $\gamma_{\mathrm{DM} i-1}, i=2, \ldots, n$ constitute the propagation constants of $\mathrm{DM}_{i-1}, i=2, \ldots, n$, respectively.

The attenuation coefficients $\alpha_{\mathrm{CM}}=\operatorname{Re}\left\{\gamma_{\mathrm{CM}}\right\}$ and $\alpha_{\mathrm{DM} i-1}=\operatorname{Re}\left\{\gamma_{\mathrm{DM} i-1}\right\}, i=2, \ldots, n$ of the CM and the $n-1$ DMs, respectively, are evaluated using the method presented in $[9,12-14,24,26,27,38,49,55-58,64]$ and are plotted versus frequency in Figures 2(a), 2(c), and 2(e) for the configurations depicted in Figures $1(\mathrm{a}), 1(\mathrm{~b})$, and $1(\mathrm{c})$, respectively. The phase delays $\beta_{\mathrm{CM}}=\operatorname{Im}\left\{\gamma_{\mathrm{CM}}\right\}$ and $\beta_{\mathrm{DM} i-1}=$ $\operatorname{Im}\left\{\gamma_{\mathrm{DM} i-1}\right\}, i=2, \ldots, n$ of the $\mathrm{CM}$ and the $n-1 \mathrm{DMs}$, respectively, [9, 12-14, 24, 26, 27, 38, 49, 55-58, 64] are also plotted versus frequency in Figures 2(b), 2(d), and 2(f) for the same three configurations, respectively.

The above modes excited-each with its own propagation characteristics - may be examined separately across the overall overhead HV transmission network, under the following three assumptions $[14,24,26-28,31,36]$.

(A1) Cables with identical eigenmodes are used throughout the network. The branches and termination points are perfectly balanced ensuring that there is no mode mixing anywhere in the network.

(A2) The branching cables are identical to the distribution cables, and the mode propagation constants of all the cable segments are assumed to be the same.

(A3) The termination points behave independently of frequency since they are either ideal matches-achieved using adaptive modal impedance matching [65, 66] — or open circuit terminations.

The three assumptions were already made in the analysis of LV/BPL and MV/BPL transmission [14, 15, 26-28, 31, 36]. Because of the above assumptions, the $n$ modes supported by the overhead HV/BPL configurations are completely separate giving rise to $n$ independent transmission channels which simultaneously carry BPL signals. This complete mode separation through the entire overhead HV/BPL transmission network has also been encountered in overhead MV/BPL and LV/BPL transmission where three and four modes, respectively, exist $[14,15,24,26,27]$.

As it has already been presented in $[13,14,24,26,64]$, the modal voltages $\mathbf{V}^{m}(z)=\left[\begin{array}{lll}V_{1}^{m}(z) & \cdots & V_{n}^{m}(z)\end{array}\right]^{T}$ and the modal currents $\mathbf{I}^{m}(z)=\left[\begin{array}{llll}I_{1}^{m}(z) & \cdots & I_{n}^{m}(z)\end{array}\right]^{T}$ may be related to the respective line quantities $\mathbf{V}(z)=\left[\begin{array}{llll}V_{1}(z) & \cdots & V_{n}(z)\end{array}\right]^{T}$ and $\mathbf{I}(z)=$ $\left[\begin{array}{llll}I_{1}(z) & \cdots & I_{n}(z)\end{array}\right]^{T}$ via the similarity transformations $[24,26,28$, 32, 33]:

$$
\begin{gathered}
\mathbf{V}(z)=\mathbf{T}_{V} \cdot \mathbf{V}^{m}(z), \\
\mathbf{I}(z)=\mathbf{T}_{I} \cdot \mathbf{I}^{m}(z),
\end{gathered}
$$

where $[\cdot]^{T}$ denotes the transpose of a matrix, $\mathbf{T}_{V}$ and $\mathbf{T}_{I}$ are $n \times n$ matrices depending on the frequency, the overhead $\mathrm{HV}$ power grid type, the geometry of the MTL configuration, and the physical properties of the cables $[9,12,13,24,26$, 28, 32, 33, 49, 64]. Through the aforementioned equations, the line voltages and currents are expressed as appropriate superpositions of the respective modal quantities. From (1),

$$
\mathbf{V}^{m}(0)=\mathbf{T}_{V}^{-1} \cdot \mathbf{V}(0)
$$

The TM method-based on the scattering matrix formalism $[15,26,37]$ and presented analytically in [15]models the spectral relationship between $V_{i}^{m}(z), i=1, \ldots, n$ and $V_{i}^{m}(0), i=1, \ldots, n$ proposing operators $H_{i}^{m}\{\cdot\}, i=$ $1, \ldots, n$ so that

$$
\mathbf{V}^{m}(z)=\mathbf{H}^{m}\left\{\mathbf{V}^{m}(0)\right\},
$$

where

$$
\mathbf{H}^{m}\{\cdot\}=\operatorname{diag}\left\{H_{1}^{m}\{\cdot\} \cdots H_{n}^{m}\{\cdot\}\right\}
$$

is a diagonal matrix operator whose elements $H_{i}^{m}\{\cdot\}, i=$ $1, \ldots, n$ are the modal transfer functions $[14,24,26]$. Combining (1) and (5), the $n \times n$ matrix channel transfer function $\mathbf{H}\{\cdot\}$ relating $\mathbf{V}(z)$ with $\mathbf{V}(0)$ through

$$
\mathbf{V}(z)=\mathbf{H}\{\mathbf{V}(0)\}
$$

is determined from

$$
\mathbf{H}\{\cdot\}=\mathbf{T}_{V} \cdot \mathbf{H}^{m}\{\cdot\} \cdot \mathbf{T}_{V}^{-1} .
$$

Based on (5), the $n \times n$ matrix transfer function $\mathbf{H}\{\cdot\}$ of the overhead HV/BPL transmission network is determined [14, $15,24,26,29,63]$.

According to how signals are injected onto overhead HV/BPL transmission lines, two different coupling schemes exist [24]. 


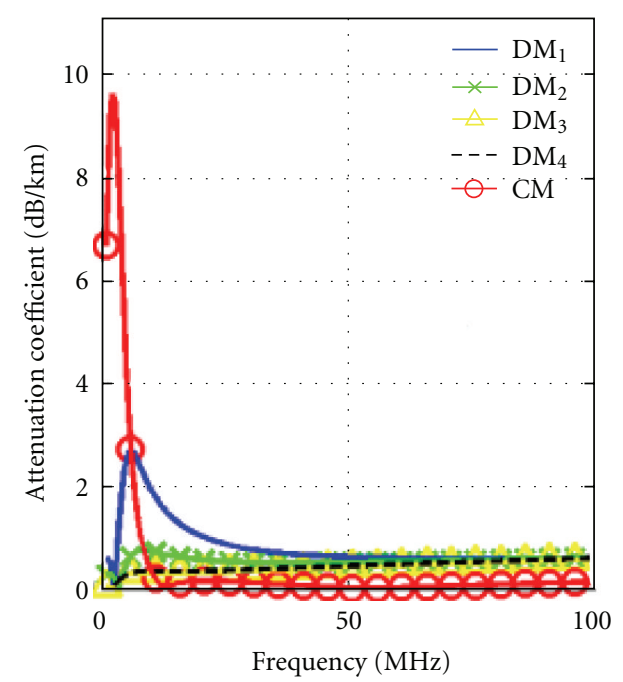

(a)

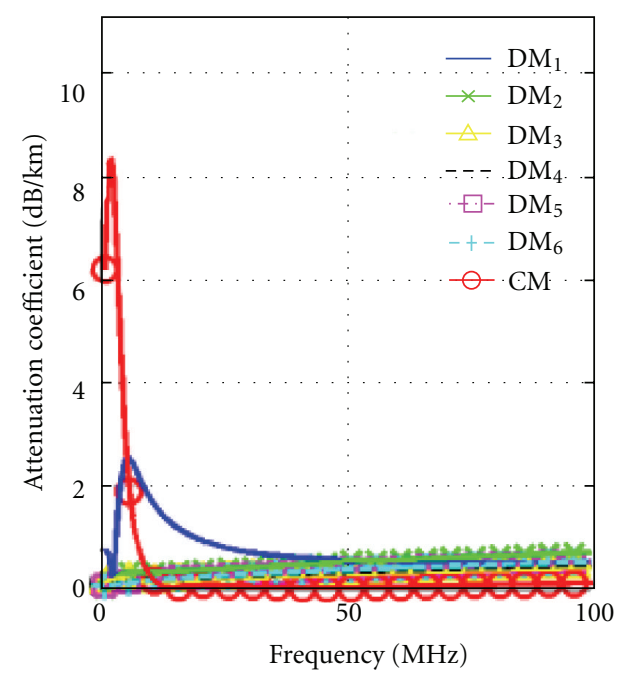

(c)

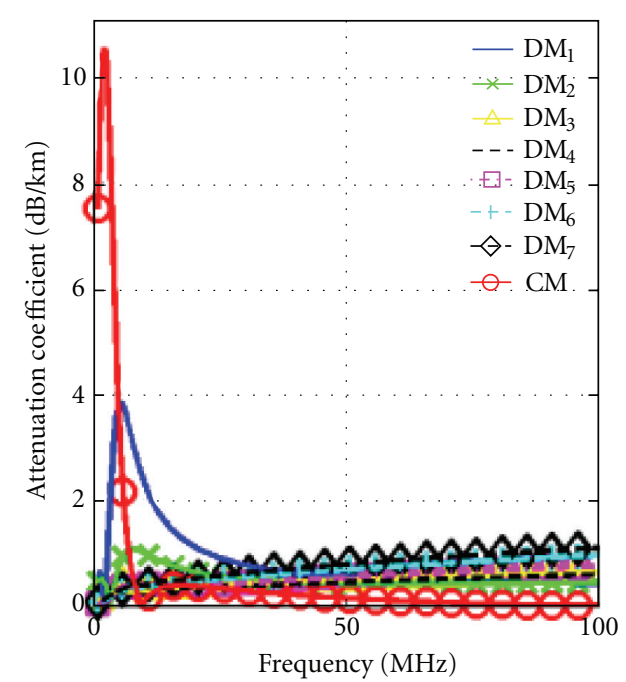

(e)

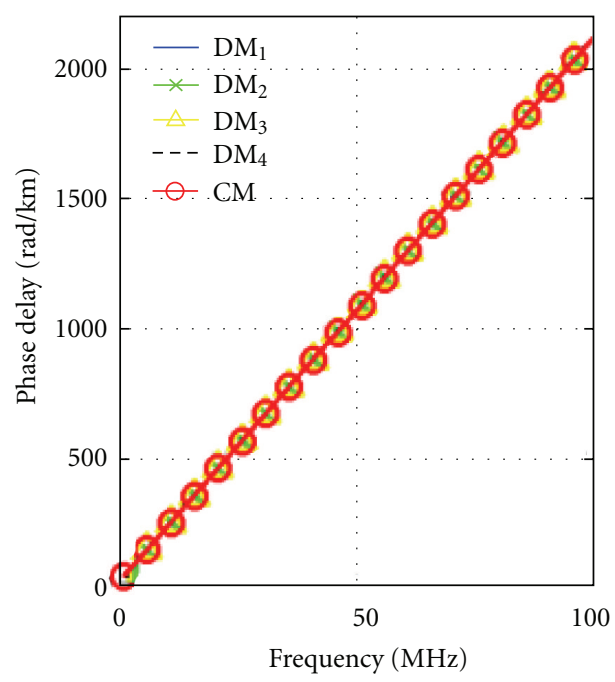

(b)

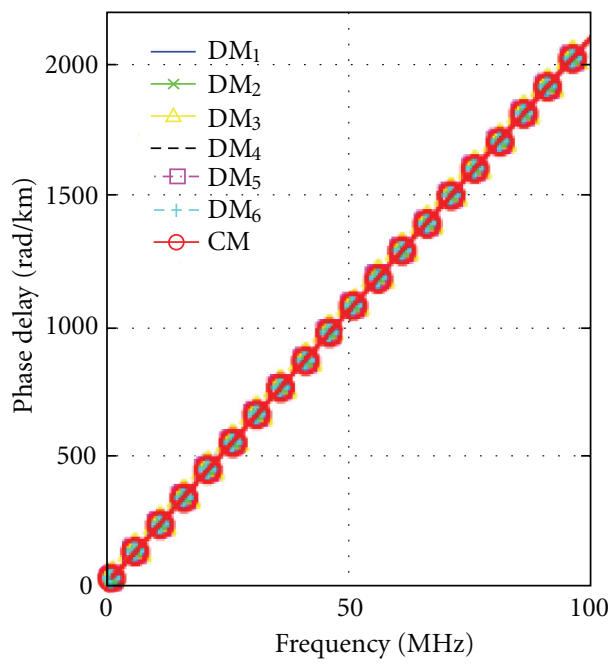

(d)

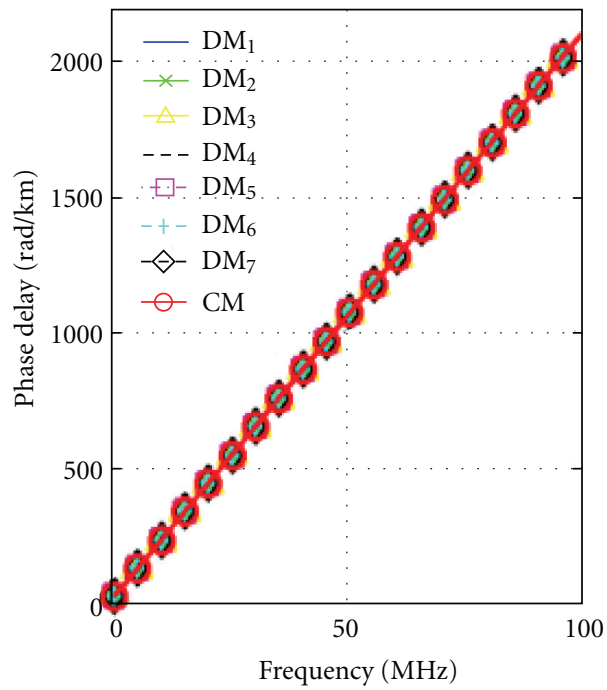

(f)

Figure 2: Frequency spectra of typical overhead HV multiconductor structures (the subchannel frequency spacing is equal to $0.1 \mathrm{MHz}$ ). (a, b) Attenuation coefficients and phase delays of $150 \mathrm{kV}$ single-circuit, respectively. (c, d) Attenuation coefficients and phase delays of $275 \mathrm{kV}$ double-circuit, respectively. (e, f) Attenuation coefficients and phase delays of $400 \mathrm{kV}$ double-circuit, respectively. 
(i) The first one is wire-to-wire (WtW) when the signal is injected between two conductors; say between conductors $p$ and $q \neq p, p, q=1, \ldots, n$. For the WtW coupling configurations, the relative excitation voltage relationship which is applied to the $n$ conductors at $z=0$ is given by

$$
\mathbf{V}(0)=V^{\mathrm{WtW}}(0) \cdot \mathbf{C}^{\mathrm{WtW}}
$$

where $V^{\mathrm{WtW}}(0)$ is the source equivalent Thévenin dipole voltage and $\mathrm{C}^{\mathrm{WtW}}$ is the $n \times 1 \mathrm{WtW}$ coupling column vector with zero elements except in rows $p$ and $q$ where the values are equal to 0.5 and -0.5 , respectively [38]. Following the same procedure, the load equivalent Thévenin dipole voltage $V^{\mathrm{WtW}}(z)$ is given from

$$
V^{\mathrm{WtW}}(z)=\left[\mathrm{C}^{\mathrm{WtW}}\right]^{T} \cdot \mathbf{V}(z)
$$

Combining (6), (7), (8), and (9), the coupling WtW transfer function $H^{\mathrm{WtW}}\{\cdot\}$ is determined by

$$
H^{\mathrm{WtW}}\{\cdot\}=\left[\mathbf{C}^{\mathrm{WtW}}\right]^{T} \cdot \mathbf{T}_{V} \cdot \mathbf{H}^{m}\{\cdot\} \cdot \mathbf{T}_{V}^{-1} \cdot \mathbf{C}^{\mathrm{WtW}} .
$$

WtW coupling between conductors $p$ and $q$ will be detoned as $\mathrm{WtW}^{p-q}$, hereafter.

(ii) The second is wire-to-ground (WtG) when the signal is injected onto one conductor and returns via the ground; say between conductor $s, s=1, \ldots, n$ and the ground. Similar expressions with (10) may be derived in WtG coupling configurations. The coupling $\mathrm{WtG}$ transfer function $H^{\mathrm{WtG}}\{\cdot\}$ is given from

$$
H^{\mathrm{WtG}}\{\cdot\}=\left[\mathbf{C}^{\mathrm{WtG}}\right]^{T} \cdot \mathbf{T}_{V} \cdot \mathbf{H}^{\mathrm{m}}\{\cdot\} \cdot \mathbf{T}_{V}^{-1} \cdot \mathbf{C}^{\mathrm{WtG}},
$$

where $\mathrm{C}^{\mathrm{WtG}}$ is the $n \times 1 \mathrm{WtG}$ coupling column vector with zero elements except in row $s$ where the value is equal to 1 [38]. WtG coupling between conductor $s$ and ground will be detoned as $\mathrm{WtG}^{s}$, hereafter.

When WtW injection is done, the DMs are mainly excited, whereas the primary excitation of the CM is generated due to the lack of symmetry of the MTL configuration; hence, BPL transmission is accomplished mostly via the DMs. When WtG injection is applied, both the CM and DMs are excited $[1,14,15,24,26,27,38,56]$.

\section{Statistical Performance Metrics of Overhead HV/BPL Channels}

Based on the end-to-end transfer functions $H\{\cdot\}$ of BPL transmission channels-either modal or coupling scheme transfer functions-several useful metrics are reported concerning the statistical properties of the respective overhead HV/BPL channels, namely, as follows.

(a) The Discrete Impulse Response. Once the end-toend transfer function is known, discrete impulse response $h_{p}=h\left(t=p T_{\mathrm{s}}\right), p=0, \ldots, J-1$ is obtained as the power of two $J$-point inverse discrete Fourier transform (IDFT) of the discrete end-to-end transfer function:

$$
\begin{aligned}
H_{q} & =\left\{\begin{array}{cc}
\left|H_{q}\right| e^{j \varphi_{q}}, & q=0, \ldots, K-1 \\
0, & q=K, \ldots, J-1
\end{array}\right\} \\
& =\left\{\begin{array}{cc}
H\left(f=q f_{s}\right), & q=0, \ldots, K-1 \\
0, & q=K, \ldots, J-1
\end{array}\right\},
\end{aligned}
$$

where $F_{\mathrm{s}}=1 / T_{\mathrm{s}}$ is the sampling rate, $f_{\mathrm{s}}$ is the flat-fading subchannel frequency spacing, $K \leq J / 2$ is the number of subchannels in the BPL signal frequency range of interest, and $\left|H_{q}\right|$ and $\phi_{q}$ are the amplitude response and the phase response of the discrete end-to-end transfer function, respectively [39-41].

(b) The Average End-to-End Channel Gain and Attenuation. As the BPL channel is frequency selective, the average endto-end channel gain can be calculated by averaging over frequency:

$$
\overline{|H|^{2}}=\sum_{p=0}^{J-1}\left|h_{p}\right|^{2}=\frac{1}{J} \sum_{q=0}^{J-1}\left|H_{q}\right|^{2},
$$

where $\overline{|H|^{2}}$ is the average end-to-end channel gain. The lognormality (normality) of the average end-to-end channel gain may be justified by the multipath nature of BPL signal propagation and the TL modeling using on cascaded two-port network modules [39-45]. In this paper, similar expression is derived in the case of average end-to-end channel attenuation $A$ by simply substituting $\overline{|H|^{2}}$ with $1 /|\bar{A}|$ in (13).

(c) The RMS-DS. It is a measure of the multipath richness of a BPL channel. The RMS-DS is determined from [39-45]

$$
\sigma_{\tau}=T_{s} \sqrt{\mu_{0}^{(2)}-\left(\mu_{0}\right)^{2}}
$$

where

$$
\begin{gathered}
\mu_{0}=\frac{\sum_{p=0}^{J-1} p\left|h_{p}\right|^{2}}{\sum_{p=0}^{J-1}\left|h_{p}\right|^{2}}, \\
\mu_{0}^{(2)}=\frac{\sum_{p=0}^{J-1} p^{2}\left|h_{p}\right|^{2}}{\sum_{p=0}^{J-1}\left|h_{p}\right|^{2}} .
\end{gathered}
$$

(d) The CB. It is the range of frequencies over which the normalized autocorrelation function of the end-to-end transfer function is over a certain CB correlation level $X$ (usually set to $0.9,0.7$, or 0.5 ), that is, a bandwidth in which the subchannels can be approximately considered flat fading. As the phase response of the end-to-end transfer function 
may be assumed as uniformly distributed over $[0,2 \pi]$, the $\mathrm{CB}$ can be determined from $[46,47]$

$$
\begin{array}{r}
B_{c}(\Delta f)=\frac{E\left\{H_{q} \cdot\left[H\left(f=q f_{\mathrm{s}}+\Delta f\right)\right]^{*}\right\}}{E\left\{\left|H_{q}\right|^{2}\right\}}, \\
q=0,1, \ldots,\left\lfloor(J-2)+\frac{\Delta f}{f_{\mathrm{s}}}\right\rfloor,
\end{array}
$$

where $\Delta f$ is the frequency shift, $[\cdot]^{*}$ denotes the complex conjugate of an element, and $\lfloor x\rfloor$ is the largest integer not greater than $x$. From (16), $\mathrm{CB}_{X}$ is that value of $\Delta f$ such that $B_{c}(\Delta f)=X$.

\section{Numerical Results and Discussion}

The simulations of various overhead HV/BPL transmission channels aim at investigating in the frequency band 1$100 \mathrm{MHz}$ : (a) their broadband transmission characteristics and how these are affected by the overhead grid topology features and (b) their inherent statistical properties. As mentioned in Section 2, since the modes supported by the overhead HV/BPL configurations may be examined separately, it is assumed for simplicity that the BPL signal is injected directly into the modes $[14,15,24,26-30,33-$ $36,38]$; thus, the complicated modal analysis of [32, 33], briefly described in Section 2, is avoided.

As it concerns the system specifications, the sampling rate and flat-fading subchannel frequency spacing are assumed $F_{\mathrm{s}}=200 \mathrm{MHz}$ and $f_{\mathrm{s}}=0.2 \mathrm{MHz}$, respectively. Thus, the number of subchannels $K$ in the BPL signal frequency range of interest and the $J$-point IDFT are assumed equal to 496 and 1024 , respectively.

For the numerical computations, the $150 \mathrm{kV}$ singlecircuit, the $275 \mathrm{kV}$ double-circuit, and the $400 \mathrm{kV}$ doublecircuit overhead HV transmission line configurations, depicted in Figures 1(a), 1(b), and 1(c), respectively, have been considered. As previously mentioned, the modes supported by the overhead HV/BPL cable configuration may be examined separately.

\subsection{Factors Influencing Overhead HV/BPL Transmission.} The following discussion will focus on the transmission characteristics related to (i) the CM and the DMs of the aforementioned overhead HV/BPL configurations and (ii) the $\mathrm{WtW}$ and the WtG coupling schemes related to these overhead HV/BPL systems as well.

The simple overhead HV/BPL topology of Figure 3, having $N$ branches, has been considered. With reference to Figure 3 , the transmitting and the receiving ends are assumed matched to the characteristic impedance of the mode considered, whereas the branch terminations $Z_{b k}, k=$ $1, \ldots, N$ are assumed open circuit $[2,13-15,24,26,27,49$, $50,64]$.

Today, thousands of $\mathrm{km}$ of overhead HV lines are installed in more than 120 countries. These lines stretch from approximately $25 \mathrm{~km}$ to $190 \mathrm{~km}$ from the generation points before reaching any population centers. Shorter branches in the range of $10 \mathrm{~km}$ to $50 \mathrm{~km}$ are used in order to connect overhead HV transmission lines either between them or with HV/MV substations [2, 13, 25, 49, 50, 64, 67, 68].

To compare the modal channels with the coupling scheme channels, a relatively dense overhead HV/BPL network of path length up to $25 \mathrm{~km}$ is assumed. The following representative overhead HV/BPL topology has been examined-see Figure 3.

(1) The "LOS" transmission along the average end-toend distance $L=L_{1}+\cdots+L_{N+1}=25 \mathrm{~km}$ when no branches are encountered. This topology corresponds to line-of-sight transmission in wireless channels.

In Figures 4(a), 4(c), and 4(e), the end-to-end channel attenuation from $\mathrm{A}$ to $\mathrm{B}$ is plotted versus frequency for the "LOS" transmission case for the propagation of CM and $\mathrm{DM}_{i-1}, i=2, \ldots, n$ for the overhead HV/BPL configurations of Figures 1(a), 1(b), and 1(c), respectively. In Figure 4(b), among the possible $n \cdot(n-1) \mathrm{WtW}$ and $n \mathrm{WtG}$ configurations, the end-to-end coupling channel attenuation from A to B for the "LOS" transmission case is plotted versus frequency in the case of overhead HV/BPL configuration of Figure 1(a) for the coupling schemes $\mathrm{WtW}^{1-2}, \mathrm{WtW}^{2-1}$, $\mathrm{WtW}^{1-3}, \mathrm{WtW}^{5-2}, \mathrm{WtG}^{1}$, and $\mathrm{WtG}^{4}$. In Figures $4(\mathrm{~d})$ and $4(\mathrm{f})$, similar curves are plotted in the case of overhead HV/BPL configuration of Figure 1(b) - coupling schemes $\mathrm{WtW}^{1-2}, \mathrm{WtW}^{1-4}, \mathrm{WtW}^{4-1}, \mathrm{WtW}^{7-3}, \mathrm{WtG}^{1}$, and $\mathrm{WtG}^{7}-$ and Figure $1(\mathrm{c})$-coupling schemes $\mathrm{WtW}^{1-2}, \mathrm{WtW}^{1-4}$, $\mathrm{WtW}^{4-1}, \mathrm{WtW}^{8-3}, \mathrm{WtG}^{1}$, and $\mathrm{WtG}^{7}-$, respectively.

From Figures 4(a), 4(b), 4(c), 4(d), 4(e), and 4(f), several interesting remarks may be highlighted.

(i) The common modes between the three overhead $\mathrm{HV} / \mathrm{BPL}$ configurations examined-CM and $\mathrm{DM}_{i-1}$, $i=2,3,4,5$-are characterized by relevant spectral behaviors-see Figures 4(a), 4(c), and 4(e). This inherent modal similarity of overhead HV/BPL systems is reflected on similar plots regarding spectral behavior of $\mathrm{WtW}$ and $\mathrm{WtG}$ coupling schemes-see Figures 4(b), 4(d), and 4(f).

(ii) As it has already been mentioned, it is confirmed that $\mathrm{WtW}$ coupling schemes are primarily affected by the propagation of DMs, whereas WtG coupling schemes are influenced mostly by CM.

(iii) Observing $\mathrm{WtW}^{1-2}$ and $\mathrm{WtW}^{2-1}$ curve plots from Figure 4(b) and $\mathrm{WtW}^{1-4}$ andWtW ${ }^{4-1}$ curve plots from Figures 4(d) and 4(f), it is clearly shown that the isotropy that characterizes other $\mathrm{MV} / \mathrm{BPL}$ and LV/BPL channels—presented in $[25,31,49,64,67]$ is also validated in overhead HV/BPL systems.

(iv) Although WtG coupling schemes present better average attenuation characteristics in comparison with WtW coupling schemes, two important factors that should be taken under consideration for appropriately evaluating HV/BPL coupling systems are (i) the significant EMI of WtG coupling schemes to other already licensed wireless communications $[3,69,70]$ 


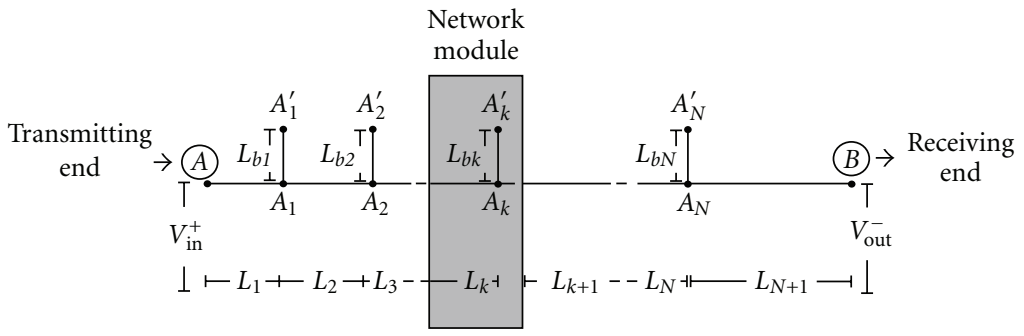

Figure 3: End-to-end HV/BPL connection with $N$ branches and a network module [15, 26].

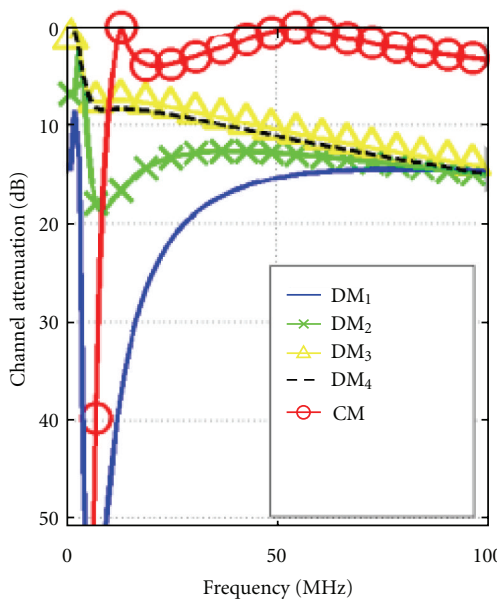

(a)

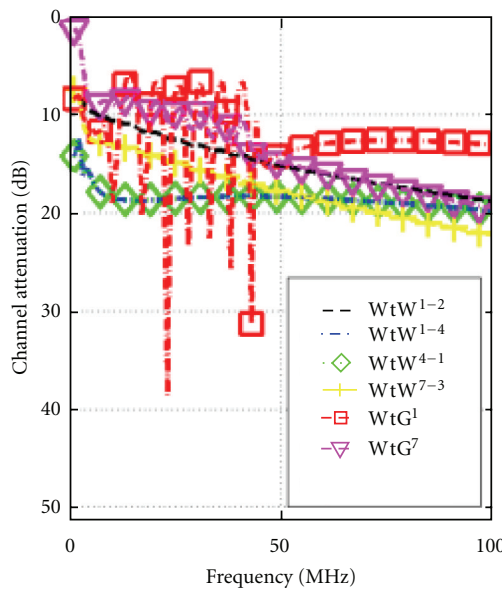

(d)

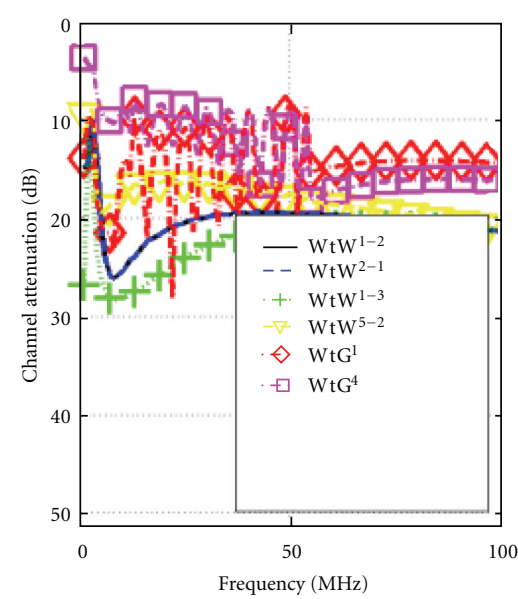

(b)

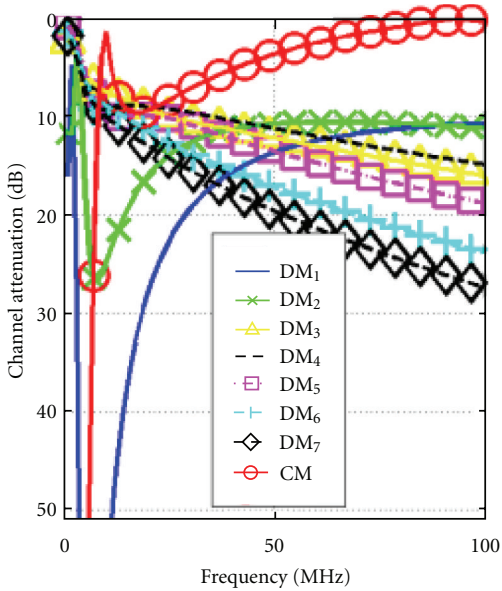

(e)

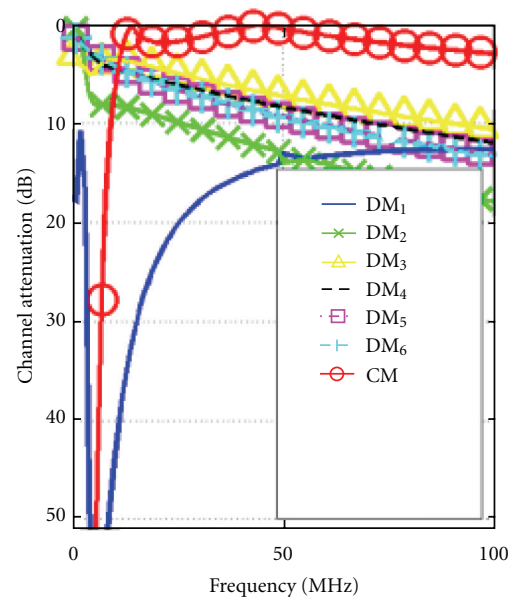

(c)

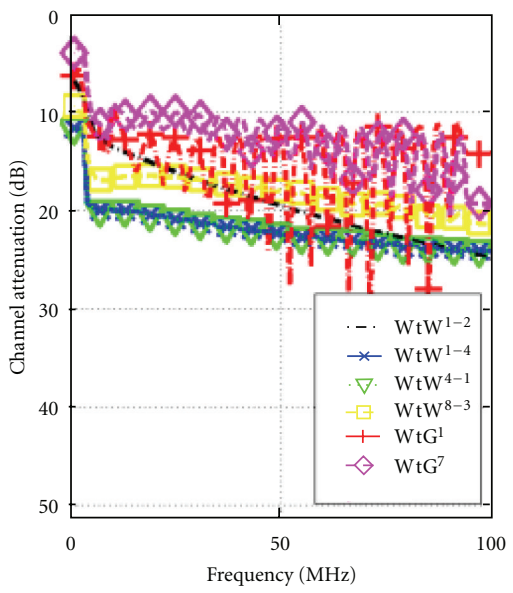

(f)

FIGURE 4: End-to-end channel attenuation versus frequency for "LOS" transmission case, (for plot clarity reasons, the subchannel frequency spacing is equal to $1 \mathrm{MHz}$ ). (a, b) Modal and coupling scheme analysis of $150 \mathrm{kV}$ single-circuit, respectively. (c, d) Modal and coupling scheme analysis of $275 \mathrm{kV}$ double-circuit, respectively. (e, f) Modal and coupling scheme analysis of $400 \mathrm{kV}$ double-circuit, respectively.

and (ii) the spectral notches that are observed in the end-to-end channel attenuation of WtG coupling schemes due to CMs. Therefore, an interesting tradeoff between EMI protection, frequency selective behavior, and channel capacity occurs during the suitable coupling scheme selection [14, 15, 47].

(v) The "LOS" transmission channels present low-loss characteristics at frequencies ranging from $1 \mathrm{MHz}$ to
$100 \mathrm{MHz}$ over a $25 \mathrm{~km}$ repeater span regardless of the overhead HV power grid type considered. The fact that overhead HV/BPL lines resemble a lowloss transmission system shows as an attractive broadband last mile solution [13-15, 24, 26-28, 34, $38,50]$.

As it has been shown, the overhead HV/BPL "LOS" configurations tend to present attenuation characteristics 
comparable to the fiber-optic ones. Practically, this favorable attenuation behavior still exists in real overhead HV topologies. With reference to Figure 3, four indicative overhead HV topologies concerning end-to-end connections of average lengths equal to $25 \mathrm{~km}$ are examined. These topologies, which are common for $150 \mathrm{kV}$ single-circuit, $275 \mathrm{kV}$ doublecircuit, and $400 \mathrm{kV}$ double-circuit overhead HV/BPL systems, are the "LOS" transmission topology referred to above and in $[2,13,49,50,64,67,68]$ :

(2) a typical urban topology (urban case) with $N=3$ branches $\left(L_{1}=1.15 \mathrm{~km}, L_{2}=12.125 \mathrm{~km}, L_{3}=8.425\right.$ $\mathrm{km}, L_{4}=3.3 \mathrm{~km}, L_{b 1}=27.6 \mathrm{~km}, L_{b 2}=17.2 \mathrm{~km}$, $\left.L_{b 3}=33.1 \mathrm{~km}\right)$,

(3) a typical suburban topology (suburban case) with $N=2$ branches $\left(L_{1}=9.025 \mathrm{~km}, L_{2}=12.75 \mathrm{~km}\right.$, $\left.L_{3}=3.225 \mathrm{~km}, L_{b 1}=46.8 \mathrm{~km}, L_{b 2}=13.4 \mathrm{~km}\right)$,

(4) a typical rural topology (rural case) with only $N=1$ branch $\left(L_{1}=3.75 \mathrm{~km}, L_{2}=21.25 \mathrm{~km}, L_{b 1}=21.1\right.$ $\mathrm{km})$.

As usually done to simplify the analysis and due to relatively comparable results among modal and coupling scheme channels - as it concerns the end-to-end channel attenuation of the "LOS" transmission case from Figures 4(a), 4(b), 4(c), $4(\mathrm{~d}), 4(\mathrm{e})$, and $4(\mathrm{f})-[5,6,14-16,24,27,38,71]$, only one mode - say $\mathrm{DM}_{4}$ - and only one coupling schemesay $\mathrm{WtW}^{1-2}$-for the modal and coupling scheme analysis, respectively, of each overhead HV/BPL system configuration of Figures 1(a), 1(b), and 1(c) will be examined, hereafter. This assumption does not affect the generality of the analysis concerning the transmission characteristics of the examined overhead $\mathrm{HV} / \mathrm{BPL}$ topologies in the range from $1 \mathrm{MHz}$ to $100 \mathrm{MHz}$, provides a representative picture of the real-world overhead HV/BPL network situation, and is adopted for the sake of terseness and simplicity.

In Figures 5(a) and 5(b), the end-to-end channel attenuation from A to B of overhead HV/BPL configuration of Figure 1(a) is plotted with respect to frequency for the aforementioned four indicative topologies for the propagation of $\mathrm{DM}_{4}$ and $\mathrm{WtW}{ }^{1-2}$, respectively. Similar plots are also plotted in Figures 5(c) and 5(d) in the case of overhead HV/BPL configuration of Figure 1(b) and in Figures 5(e) and 5(f) in the case of overhead HV/BPL configuration of Figure 1(c).

According to the picture obtained from their spectral behavior-see Figures 5(a), 5(b), 5(c), 5(d), 5(e), and 5(f), the overhead HV/BPL topologies may be classified into three major channel classes regardless of the overhead HV/BPL channel type examined-either modal or coupling scheme channel-(see also $[14,15,24,71]$ for other MV/BPL and LV/BPL channels)

(1) The firt one is "LOS" channels, when no branches are encountered, and, consequently, no spectral notches are observed. This case corresponds to the best possible overhead HV/BPL transmission conditions, encountered when long-distance transmission occurs.
(2) The second is good channels, when the number of branches is small and their electrical length is large. Shallow spectral notches are observed. Overhead HV/BPL transmission primarily near rural and suburban areas belongs to this channel class.

(3) The third is bad channels, when the number of branches is large and their electrical length is small. Deep spectral notches are observed. Overhead HV/BPL transmission near urban areas belongs to this channel class.

Thus, the spectral behavior of the above overhead HV/BPL channel classes affects critically the transmission characteristics of overhead HV/BPL channels [10, 14, 15, 22, 24, 26, 7274].

4.2. On the Statistical Relationship between Performance Metrics in Overhead HV/BPL Transmission. Based on the statistical performance metrics presented in Section 4, a physically meaningful statistical characterization of the BPL channels is obtained permitting important properties of the overhead HV/BPL systems to be revealed. Tables 1, 2, and 3 summarize the metrics of average end-to-end channel attenuation, RMS-DS, and $\mathrm{CB}_{0.5}$, respectively, for the propagation of $\mathrm{DM}_{4}$ and $\mathrm{WtW}^{1-2}$ for $150 \mathrm{kV}$ single-circuit, $275 \mathrm{kV}$ double-circuit, and $400 \mathrm{kV}$ double-circuit overhead HV/BPL configurations for each of the aforementioned four indicative HV/BPL topologies.

Observations of Tables 1, 2, and 3 demonstrate that the values of performance metrics validate the above overhead HV/BPL channel classification. Analytically, the metrics of average end-to-end channel attenuation of "LOS" and good channel class transmission are significantly better than the bad ones. Furthermore, an indicative picture of the multipath aggravation that affects primarily the bad channel class transmission can be obtained studying the metrics of RMS-DS and CB [39-47].

Based on the statistical performance metrics of Tables 1,2 , and 3, fundamental properties of several wireline systems (e.g., DSL, coaxial, and phone links) can be also validated in the overhead HV/BPL system case. First, the relationship between the channel attenuation and RMS-DS is investigated. The correlation between average end-to-end channel attenuation and RMS-DS can be easily confirmed observing the scatter plot presented in Figure 6 where the above set of numerical results of $\mathrm{DM}_{4}$ and $\mathrm{WtW}{ }^{1-} 2$ for the aforementioned indicative overhead HV/BPL topologiesas presented analytically in Tables 1 and 2 and denoted as overhead HV/BPL power networks in Figure 6-are plotted. In Figure 6, apart from these numerical results, the following sets of field trial measurements and regression trend lines are also presented:

(i) measurements in real MV/BPL networks: (i) underground MV/BPL power network [39-41] and (ii) test MV/BPL power network [43],

(ii) measurements in real LV/BPL networks: (i) in-home LV/BPL suburban and urban networks $[75,76]$ and 


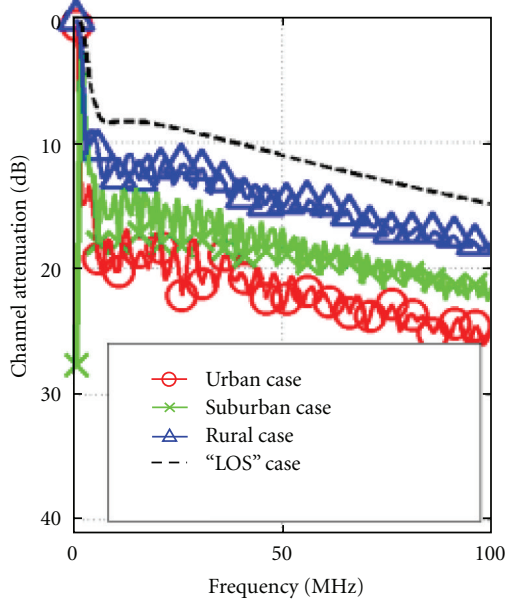

(a)

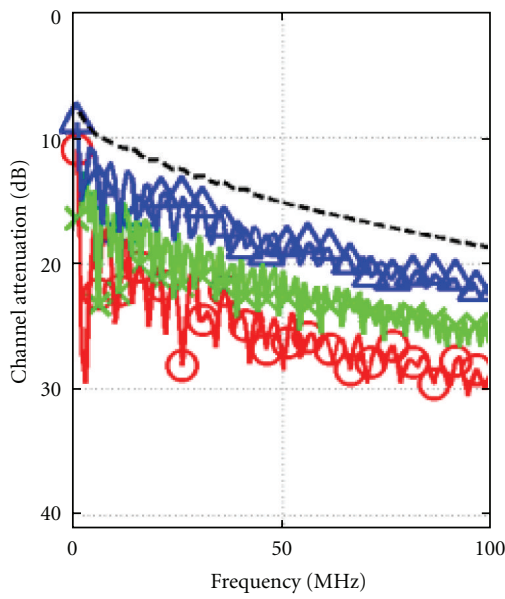

(d)

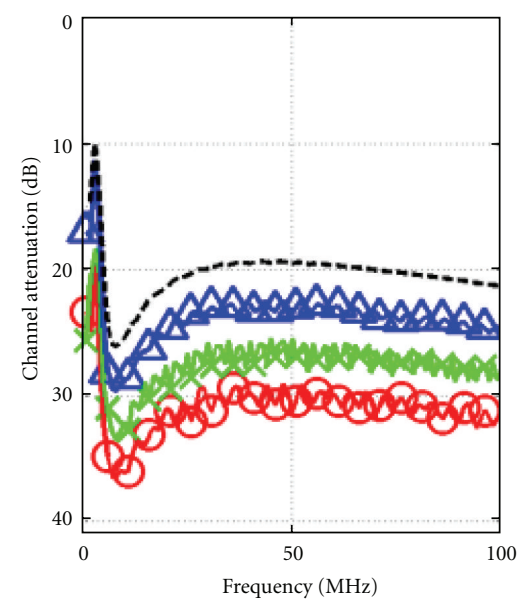

(b)

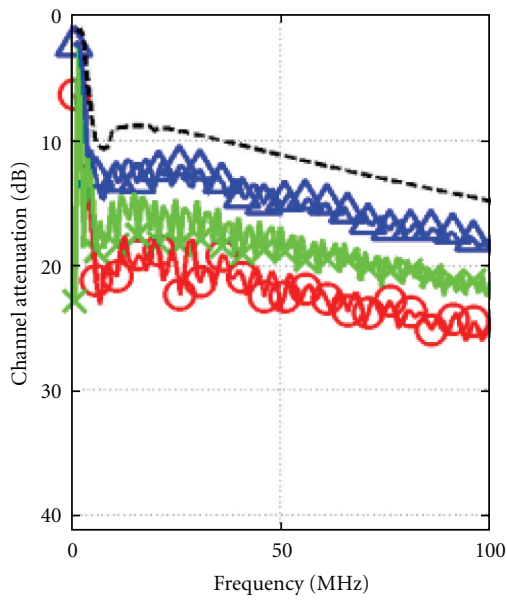

(e)

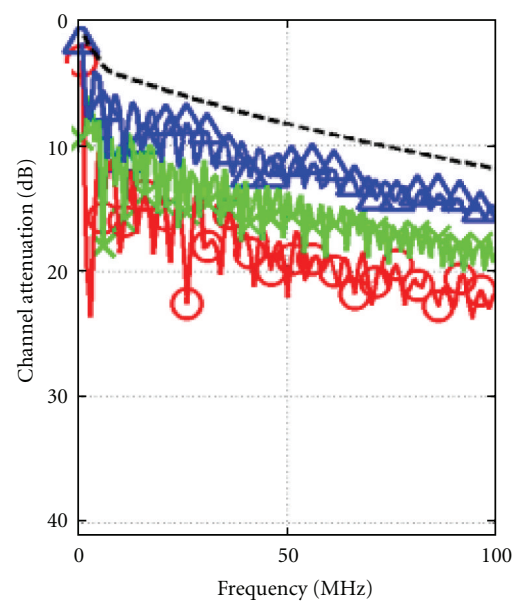

(c)

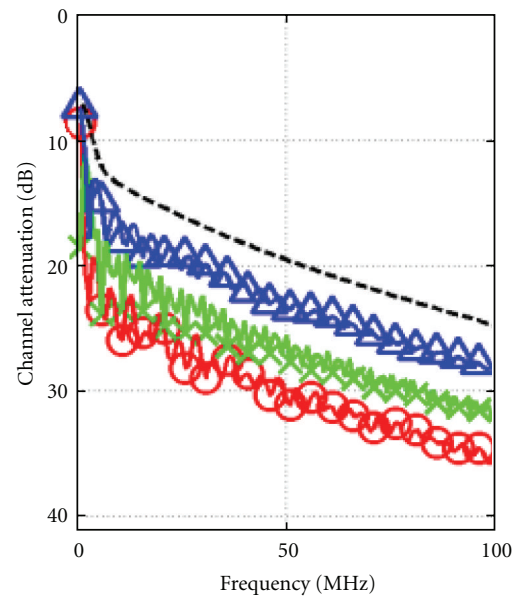

(f)

FIGURE 5: End-to-end channel attenuation versus frequency for urban case, suburban case, rural case, and "LOS" transmission case when open circuit branch terminations are assumed (for plot clarity reasons, the subchannel frequency spacing is equal to $1 \mathrm{MHz}$ ). (a, b) Modal$\mathrm{DM}_{4}$ - and coupling scheme-WtW ${ }^{1-2}$ - analysis of $150 \mathrm{kV}$ single-circuit, respectively. (c, d) Modal—DM 4 -and coupling scheme$\mathrm{WtW}^{1-2}$ — analysis of $275 \mathrm{kV}$ double-circuit, respectively. (e, f) Modal- $\mathrm{DM}_{4}$ —and coupling scheme-WtW ${ }^{1-2}$-analysis of $400 \mathrm{kV}$ doublecircuit, respectively.

TABLE 1: Average end-to-end channel attenuation for various overhead HV/BPL configurations and topologies.

\begin{tabular}{|c|c|c|c|c|c|c|}
\hline & \multicolumn{6}{|c|}{ Average end-to-end channel attenuation (dB) } \\
\hline & \multicolumn{2}{|c|}{$150 \mathrm{kV}$} & \multicolumn{2}{|c|}{$275 \mathrm{kV}$} & \multicolumn{2}{|c|}{$400 \mathrm{kV}$} \\
\hline & $\mathrm{DM}_{4}$ & $\mathrm{WtW}^{1-2}$ & $\mathrm{DM}_{4}$ & $\mathrm{WtW}^{1-2}$ & $\mathrm{DM}_{4}$ & $\mathrm{WtW}^{1-2}$ \\
\hline Urban case & 21.82 & 32.41 & 20.06 & 26.73 & 23.25 & 29.48 \\
\hline Suburban case & 19.30 & 29.91 & 16.86 & 23.47 & 19.84 & 26.37 \\
\hline Rural case & 16.08 & 26.36 & 13.65 & 20.23 & 16.43 & 23.14 \\
\hline "LOS" case & 12.96 & 22.90 & 10.43 & 16.98 & 13.22 & 19.98 \\
\hline
\end{tabular}

(ii) indoor LV/BPL power networks—inside homes and small offices [46],

(iii) regression lines of the form $\left(\sigma_{\tau}\right)_{\mu \mathrm{s}}=v \cdot(\bar{A})_{\mathrm{dB}}+w$ where $(\bar{A})_{\mathrm{dB}}$ is the average end-to-end channel attenuation in $\mathrm{dB}$ and $\left(\sigma_{\tau}\right)_{\mu \mathrm{s}}$ is the RMS-DS in $\mu$ s of the BPL channel examined: (i) GAL approach, as given by [39-41], with robust regression parameters assumed $v=0.0075 \mu \mathrm{s} / \mathrm{dB}$ and $w=0.183 \mu \mathrm{s}$; (ii) TON approach, as given by [43], with robust regression parameters $v$ and $w$ assumed equal to $0.0197 \mu \mathrm{s} / \mathrm{dB}$ and $0 \mu \mathrm{s}$, respectively; (iii) the proposed $\mathrm{UNI}^{1}$ approach with regression parameters $v=$ $0.0129 \mu \mathrm{s} / \mathrm{dB}$ and $w=0.2472 \mu \mathrm{s}$. The least squares 
TABLE 2: RMS-DS for various overhead HV/BPL configurations and topologies.

\begin{tabular}{lcccccc}
\hline & \multicolumn{2}{c}{ RMS-DS $(\mu \mathrm{s})$} & \multicolumn{2}{c}{$400 \mathrm{kV}$} \\
& \multicolumn{2}{c}{$150 \mathrm{kV}$} & \multicolumn{2}{c}{$275 \mathrm{kV}$} & $\mathrm{DM}_{4}$ & $\mathrm{WtW}_{4}$ \\
& $\mathrm{DM}_{4}$ & $\mathrm{WtW}^{1-2}$ & 0.36 & 0.33 & 0.84 & 0.49 \\
\hline Urban case & 1.12 & 0.80 & 0.40 & 0.36 & 0.73 & 0.48 \\
Suburban case & 0.66 & 0.62 & 0.24 & 0.23 & 0.81 & 0.31 \\
Rural case & 0.79 & 0.72 & 0.10 & 0.11 & 0.65 & 0.16 \\
"LOS" case & 0.46 & 0.64 & & & & $\mathrm{WtW}^{1-2}$ \\
\hline
\end{tabular}

TABLE 3: $\mathrm{CB}_{0.5}$ for various overhead HV/BPL configurations and topologies.

\begin{tabular}{lcccccc}
\hline & \multicolumn{2}{c}{$\mathrm{CB}_{0.5}(\mathrm{kHz})$} & \multicolumn{2}{c}{$400 \mathrm{kV}$} \\
& $\mathrm{DM}_{4}$ & $150 \mathrm{kV}$ & \multicolumn{2}{c}{$275 \mathrm{kV}$} & $\mathrm{DM}_{4}$ & $\mathrm{WtW}_{4} \mathrm{tW}^{1-2}$ \\
\hline Urban case & 9400 & 17800 & 29200 & 29400 & 25400 & 17000 \\
Suburban case & 24000 & 32400 & 30800 & 30600 & 24000 & 20800 \\
Rural case & 33000 & 32000 & 37200 & 37000 & 29400 & 26400 \\
"LOS" case & 38400 & 32800 & 42600 & 40800 & 34200 & 30400 \\
\hline
\end{tabular}

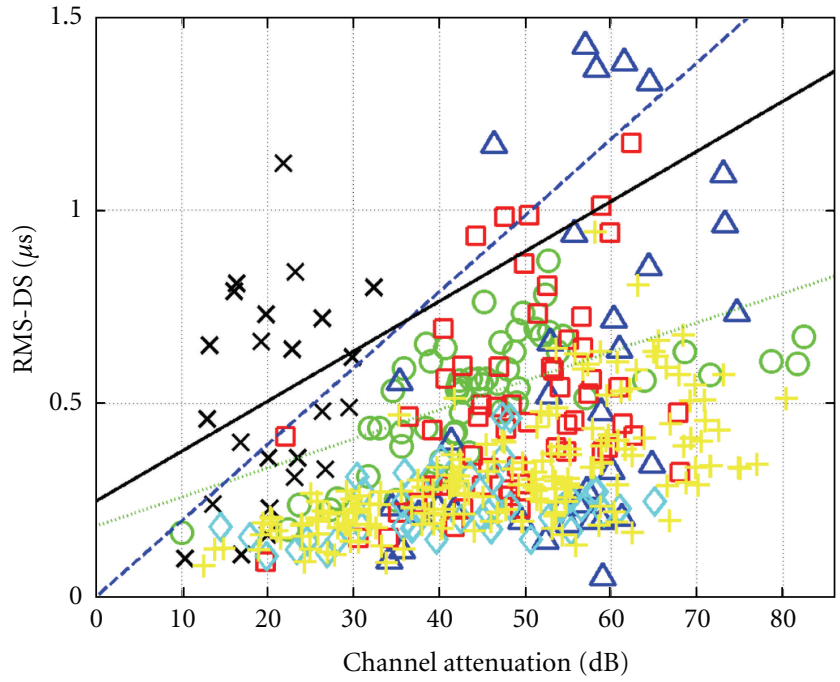

- Trend line curves-GAL

--- TON

$-\mathrm{UNI}^{1}$

Figure 6: Scatter plot of RMS-DS versus average end-to-end channel attenuation for simulated channels-overhead HV/BPL networks for the propagation of $\mathrm{DM}_{4}$ and coupling scheme $\mathrm{WtG}^{1}$ $(\times)$, measured channels - underground MV/BPL power network $(\bigcirc)$, test MV/BPL power network $(\triangle)$, in-home LV/BPL suburban networks $(\square)$, in-home LV/BPL urban networks $(\diamond)$, and indoor LV/BPL power networks $(+)$ - and trend line curves—GAL $(\cdots)$, TON (- -), and $\mathrm{UNI}^{1}(-)$ approaches-.

fitting method is applied to the aforementioned overhead HV/BPL numerical results for the evaluation of $\mathrm{UNI}^{1}$ approach parameters $v$ and $w$.

From Figure 6, the following should be mentioned. (i) All regression lines exhibit positive slopes clearly confirming that the average end-to-end channel attenuation and RMS-DS of overhead HV/BPL channels are positively correlated lognormal random variables regardless of the overhead HV power grid and channel type. This fundamental property of $\mathrm{MV} / \mathrm{BPL}, \mathrm{LV} / \mathrm{BPL}$, and several wireline channels is also validated in overhead HV/BPL case [39-47].

(ii) The proposed UNI ${ }^{1}$ trend line suggests a unified regression line that describes the correlation between channel attenuation and RMS-DS in transmission power grids. UNI ${ }^{1}$ approach offers a useful tool towards BPL/SG systems coexistence as it can be used to estimate the maximum data rate of transmission power grids being supported without the use of an equalizer.

(iii) The sets of overhead HV/BPL numerical results are well fitted by the proposed three trend lines. However, the differences between theoretical and experimental results highlight how the arbitrary choice of (i) the overhead HV power grid type, (ii) the overhead HV channel type considered, (iii) the examined overhead HV/BPL channel class, and (iv) the BPL network type in measurement campaigns, affects the statistics.

(iv) Essentially, it is demonstrated that the RMS-DS increases when the average end-to-end attenuation increases; therefore, BPL channels with large RMSDS—-severe intersymbol interference (ISI) - are also characterized by large values of end-to-end channel attenuation and, thus, low SNR for fixed transmit power.

(v) On the basis of the confirmed correlation, statistical channel models may be defined. These models can generate BPL channels either manually adjusted or stastitically assessed conferring a rather stochastic 


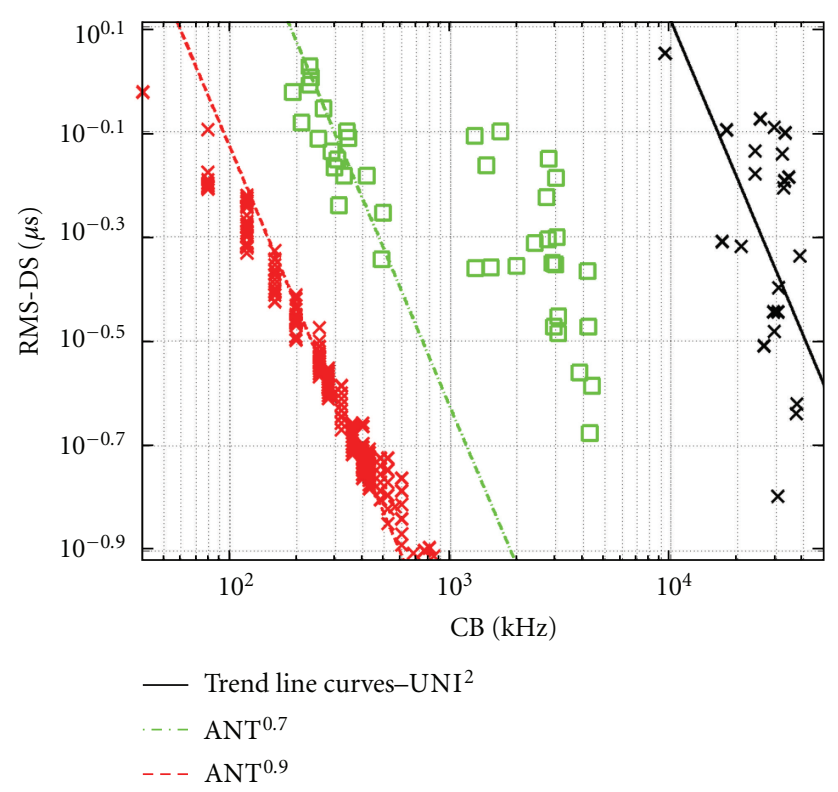

FIgURE 7: Scatter plot of RMS-DS versus CB for the simulated channels-overhead HV/BPL networks for the propagation of $\mathrm{DM}_{4}$ and coupling scheme $\mathrm{WtW}^{1-2}$ - (black $\left.\times\right)$, measured channelscruise ship LV/BPL power networks $(\square)$ and indoor LV/BPL power networks $($ red $\times)$ - and trend line curves- $\mathrm{UNI}^{2}(-), \mathrm{ANT}^{0.7}(-\cdot)$, and $\mathrm{ANT}^{0.9}(--)$ approaches (logarithmic scale was used for the $x$ and $y$-axes).

aspect to the existing deterministic channel class design [39-43, 46, 47].

Based on the statistical performance metrics of Tables 2 and 3 , the second fundamental property of several wireline systems that can be also validated in the overhead HV/BPL system case is the correlation between CB and RMS-DS. In Figure 7, the scatter plot of the RMS-DS versus the $\mathrm{CB}_{X}-$ $\mathrm{CB}_{0.9}, \mathrm{CB}_{0.7}$, and $\mathrm{CB}_{0.5}$ - is presented for the following sets of numerical results, measurements, and regression trend lines:

(i) numerical results-RMS-DS versus $\mathrm{CB}_{0.5}$ - for the overhead HV/BPL channels for the propagation of $\mathrm{DM}_{4}$ and for the coupling scheme $\mathrm{WtW}^{1-2}$-as presented in Tables 1 and 2,

(ii) field trial measurements: (i) in indoor LV/BPL power networks (inside homes and small offices)-RMSDS versus $\mathrm{CB}_{0.9}$ [46] - and (ii) in cruise ship LV/BPL power networks-RMS-DS versus $\mathrm{CB}_{0.7}$ [44],

(iii) hyperbolic trend lines of the form $\left(\sigma_{\tau}\right)_{\mu \mathrm{s}}=y /$ $\left(\mathrm{CB}_{X}\right)_{\mathrm{kHz}}$ where $\left(\mathrm{CB}_{X}\right)_{\mathrm{kHz}}$ is the $\mathrm{CB}_{X}$ in $\mathrm{kHz}$ : (i) $\mathrm{ANT}^{0.9}$ approach, as given by $[44,45,47]$, with robust regression parameter assumed $y=74 \mu \mathrm{s} \cdot \mathrm{kHz}$ that approximates the relation between RMS-DS and $\mathrm{CB}_{0.9}$, (ii) $\mathrm{ANT}^{0.7}$ approach, as given by $[44,45,47]$, with robust regression parameter $y$ assumed equal to $236 \mu \mathrm{s} \cdot \mathrm{kHz}$ that describes the relation between RMSDS and $\mathrm{CB}_{0.7}$; (iii) the proposed $\mathrm{UNI}^{2}$ approach with parameter $y$ assumed equal to $12920 \mu \mathrm{s} \cdot \mathrm{kHz}$ obtained using robust linear least square error fitting between the trend line and the observed overhead HV/BPL numerical results.

On the basis of the confirmed correlation between $\mathrm{CB}$ and RMS-DS, additional remarks are pointed out [44-47].

(i) In the first place, $\mathrm{CB}$ is a statistical measure of the range of frequencies over which the overhead HV/BPL channel can be considered to have approximately equal end-to-end attenuation and linear phase. The values of the estimated $\mathrm{CB}_{0.5}$ for overhead HV/BPL channels are ranging above $9.4 \mathrm{MHz}$, regardless of the overhead $\mathrm{HV}$ power grid type, the overhead HV channel type considered, and the examined overhead HV/BPL channel class. In the second place, the RMS-DS describes the dispersion in the time domain due to multipath transmission, and its maximum value for the overhead $\mathrm{HV} / \mathrm{BPL}$ channels examined is equal to $1.12 \mu \mathrm{s}$ that is comparable to indoor LV/BPL channel RMS-DS values. Hence, apart from low-loss attenuation characteristics that have already been mentioned, overhead HV/BPL power grid presents excellent performance as it concerns flat-fading features and multipath dispersion. In system design terms, higher $\mathrm{CB}$ values and lower multipath effects determine faster symbol transmission rates having an impact on the choice of the parameters of multicarrier systems applied over overhead HV/BPL systems [1, 40, 41, 47, 76].

(ii) The variability of the $\mathrm{CB}_{X}$ and RMS-DS depends on the $\mathrm{CB}$ correlation level, the overhead HV/BPL channel class, the topology characteristics, the overhead HV/BPL power grid type considered, and the overhead HV/BPL channel type examined. Their relation quantifies the effects of frequency selective behavior and multipath propagation environment that further affect BPL channel capacity $[14,44,48]$.

(iii) $\mathrm{CB}$ and RMS-DS are inversely related and their relation can be approximated by hyperbolic functions. $\mathrm{UNI}^{2}$ approach and the other trend line results are in excellent agreement with the simulations results and the measurements, respectively.

From the previous figures, several interesting conclusions concerning overhead $\mathrm{HV} / \mathrm{BPL}$ transmission characteristics may be deduced as follows.

(1) Though determined for $25 \mathrm{~km}$ long HV connections - compared to the shorter connections of MV and $\mathrm{LV}$ cases $[1,2,10,14-17,24,26-29,74]-\mathrm{BPL}$ transmission via the overhead $\mathrm{HV}$ grid exhibits lowloss characteristics regardless of the overhead HV power grid type favoring the exploitation of $\mathrm{HV} / \mathrm{BPL}$ bandwidth.

(2) As usually done in BPL systems [14, 15, 24, 71], overhead HV/BPL channels are classified into three classes depending on their spectral behavior: "LOS" channels, good channels, and bad channels. HV/BPL transmission in the majority of areas is classified into the good channels class. 
(3) In overhead HV/BPL channels, it is verified that channel attenuation and RMS-DS are (positively) correlated lognormal random variables [39-47]. Moreover, CB and RMS-DS are inversely related and may be approximated by hyperbolic functions [44-47]. These fundamental properties of several wireline and BPL systems (e.g., DSL, coaxial, phone, LV/BPL, and MV/BPL links) are also validated in the overhead HV/BPL system case. As it concerns these inherent statistical characteristics of overhead HV/BPL systems, suitable approximations can be proposed, like $\mathrm{UNI}^{1}$ and $\mathrm{UNI}^{2}$ regression trend lines.

(4) Observing the same characteristics of HV/BPL, MV/BPL, and LV/BPL channels-especially in Figures 6 and 7-a preliminary step towards their common analysis is introduced; through the presented hybrid method and the proposed regression approximations, BPL systems may be examined under a common PHY framework. This introduction of unified tools for the common handling of BPL transmission and distribution networks is to be seen as strong interoperability guarantee in a SG landscape.

(5) Apart from the low-loss attenuation behavior, overhead HV/BPL channels demonstrate impressive flatfading characteristics and low multipath dispersion rendering, thus, HV/BPL transmission as a fiberoptic transmission alternative solution.

(6) The deterministic BPL channel models can coexist with statistical BPL ones in order to simulate a wide variety of BPL channels and generate random classoriented BPL topologies.

(7) Concluding the above HV/BPL technology analysis, it should be highlighted that SG will be supported by a heterogeneous set of networking technologies, as no single solution fits all scenarios. HV/BPL, MV/BPL, and LV/BPL systems need to work in a compatible way (intraoperate) before BPL technology interoperates with other broadband technologies, such as wired (e.g., fiber and DSL) and wireless (e.g., WiFi and WiMax). Based on an IP-based power system, related issues to BPL technology such as compatible frequencies, equipment and signaling, adequate injected power spectral density (IPSD) levels, area coverage, and scalable capacity may be further facilitated, taking into account the specific features of $\mathrm{HV} / \mathrm{BPL}, \mathrm{MV} / \mathrm{BPL}$, and LV/BPL transmission together with the overall system requirements $[1,14$, $24,25,71]$.

\section{Conclusions}

This paper has focused on the broadband transmission characteristics and the statistical performance metrics of overhead HV/BPL transmission power grid.

The transmission characteristics of overhead HV/BPL transmission networks have been studied applying the wellknown TM method. The broadband transmission capability of such networks depends on the frequency, the overhead HV power grid type, the coupling scheme applied, the mode examined, physical properties of the MTL configuration used, the end-to-end- "LOS"-distance, and the number, the electrical length, and the terminations of the branches along the end-to-end overhead HV/BPL signal propagation.

Based on the statistical performance metrics of overhead HV/BPL transmission networks, fundamental properties of several wireline channels, that are (i) the positive correlation between end-to-end channel attenuation and RMS-DS and (ii) the hyperbolic correlation between $\mathrm{CB}$ and RMS-DS; have also been validated in the case of overhead HV/BPL systems against relevant sets of field measurements, numerical results, and statistical distributions. Based on these inherent attributes of BPL systems, new regression trend lines have been proposed. The new approximations give a further boost towards BPL system interoperability.

The low-loss nature of overhead HV/BPL systems, the impressive flat-fading characteristics, and the relatively low multipath dispersion over a $25 \mathrm{~km}$ repeater span well beyond $100 \mathrm{MHz}$ render overhead $\mathrm{HV}$ power lines as an alternative fiber-optic technology permitting the further exploitation of HV/BPL bandwidth towards a unified transmission/distribution of SG power grid.

\section{References}

[1] H. Ferreira, L. Lampe, J. Newbury, and T. G. Swart, Power Line Communications, Theory and Applications for Narrowband and Broadband Communications over Power Lines, John Wiley \& Sons, New York, NY, USA, 2010.

[2] OPERA1, "D44: report presenting the architecture of PLC system, the electricity network topologies, the operating modes and the equipment over which PLC access system will be installed," IST Integr. Project No 507667, 2005.

[3] NATO, "HF interference, procedures and tools (Interférences $\mathrm{HF}$, procédures et outils) final report of NATO RTO information systems technology," RTO-TR-ISTR-050, 2007, http://ftp.rta.nato.int/public/PubFullText/RTO/TR/RTO-TR-IST-050/\$\$TR-IST-050-ALL.pdf.

[4] R. Aquilué, I. Gutierrez, J. L. Pijoan, and G. Sánchez, "Highvoltage multicarrier spread-spectrum system field test," IEEE Transactions on Power Delivery, vol. 24, no. 3, pp. 1112-1121, 2009.

[5] R. Aquilué, J. L. Pijoan, and G. Sánchez, "High voltage channel measurements and field test of a low power OFDM system," in Proceedings of the IEEE International Symposium on Power Line Communications and Its Applications (ISPLC '08), pp. 1-6, Jeju Island, South Korea, April 2008.

[6] N. Suljanović, A. Mujčić, M. Zajc, and J. F. Tasič, "Corona noise characteristics in high voltage PLC channel," in Proceedings of the IEEE International Conference on Industrial Technology, vol. 2, pp. 1036-1039, Maribor, Slovenia, March 2003.

[7] A. Mujčić, N. Suljanović, M. Zajc, and J. F. Tasič, "Design of channel coding methods in HV PLC communications," in Proceedings of the IEEE International Symposium on Power Line Communications and Its Applications (ISPLC '04), pp. 379384, Zaragoza, Spain, March 2004.

[8] A. Mujčić, N. Suljanović, M. Zajc, and J. F. Tasič, "High-voltage PLC roles in packet-switching networks of power utilities," in 
Proceedings of the IEEE International Symposium on Power Line Communications and Its Applications (ISPLC '07), pp. 204209, Pisa, Italy, March 2007.

[9] R. Pighi and R. Raheli, "On multicarrier signal transmission for high-voltage power lines," in Proceedings of the International Symposium on Power Line Communications and Its Applications (ISPLC '05), pp. 32-36, Vancouver, Canada, April 2005.

[10] K. Dostert, Powerline Communications, Prentice Hall, Upper Saddle River, NJ, USA, 2001.

[11] N. Suljanović, A. Mujčić, M. Zajc, and J. F. Tasič, "Computation of high-frequency and time characteristics of corona noise on HV power line," IEEE Transactions on Power Delivery, vol. 20, no. 1, pp. 71-79, 2005.

[12] N. Suljanović, A. Mujčić, M. Zajc, and J. F. Tasič, "Integrated communication model of the HV power-line channel," in Proceedings of the IEEE International Symposium on Power Line Communications and Its Applications (ISPLC '04), pp. 79-84, Zaragoza, Spain, March 2004.

[13] N. Suljanović, A. Mujčić, M. Zajc, and J. F. Tasič, "Approximate computation of high-frequency characteristics for power line with horizontal disposition and middle-phase to ground coupling," Electric Power Systems Research, vol. 69, no. 1, pp. 17-24, 2004.

[14] A. G. Lazaropoulos and P. G. Cottis, "Capacity of overhead medium voltage power line communication channels," IEEE Transactions on Power Delivery, vol. 25, no. 2, pp. 723-733, 2010.

[15] A. G. Lazaropoulos and P. G. Cottis, "Broadband transmission via underground medium-voltage power lines-part II: capacity," IEEE Transactions on Power Delivery, vol. 25, no. 4, pp. 2425-2434, 2010.

[16] M. Gebhardt, F. Weinmann, and K. Dostert, "Physical and regulatory constraints for communication over the power supply grid," IEEE Communications Magazine, vol. 41, no. 5, pp. 8490, 2003.

[17] P. S. Henry, "Interference characteristics of broadband power line communication systems using aerial medium voltage wires," IEEE Communications Magazine, vol. 43, no. 4, pp. 92 98, 2005.

[18] S. Liu and L. J. Greenstein, "Emission characteristics and interference constraint of overhead medium-voltage broadband power line (BPL) systems," in Proceedings of the IEEE Global Telecommunications Conference (GLOBECOM '08), pp. 1-5, New Orleans, La, USA, November 2008.

[19] M. Götz, M. Rapp, and K. Dostert, "Power line channel characteristics and their effect on communication system design," IEEE Communications Magazine, vol. 42, no. 4, pp. 78-86, 2004.

[20] D. Fenton and P. Brown, "Some aspects of benchmarking high frequency radiated emissions from wireline communications systems in the near and far fields," in Proceedings of the IEEE International Symposium on Power Line Communications and Its Applications (ISPLC '01), pp. 161-167, Malmö, Sweden, April 2001.

[21] D. Fenton and P. Brown, "Modelling cumulative high frequency radiated interference from power line communication systems," in Proceedings of the IEEE International Symposium on Power Line Communications and Its Applications (ISPLC '02), Athens, Greece, March 2002.

[22] M. Zimmermann and K. Dostert, "A multipath model for the powerline channel," IEEE Transactions on Communications, vol. 50, no. 4, pp. 553-559, 2002.
[23] S. Galli and O. Logvinov, "Recent developments in the standardization of power line communications within the IEEE," IEEE Communications Magazine, vol. 46, no. 7, pp. 64-71, 2008.

[24] A. G. Lazaropoulos, "Broadband transmission characteristics of overhead high-voltage power line communication channels," Progress In Electromagnetics Research B, no. 36, pp. 373$398,2012$.

[25] S. Galli, A. Scaglione, and Z. Wang, "For the grid and through the grid: the role of power line communications in the smart grid," Proceedings of the IEEE, vol. 99, no. 6, pp. 998-1027, 2011.

[26] A. G. Lazaropoulos and P. G. Cottis, "Transmission characteristics of overhead medium-voltage power-line communication channels," IEEE Transactions on Power Delivery, vol. 24, no. 3, pp. 1164-1173, 2009.

[27] P. Amirshahi and M. Kavehrad, "High-frequency characteristics of overhead multiconductor power lines for broadband communications," IEEE Journal on Selected Areas in Communications, vol. 24, no. 7, pp. 1292-1303, 2006.

[28] T. Sartenaer, Multiuser communications over frequency selective wired channels and applications to the powerline access network, Ph.D. dissertation, Université Catholique de Louvain, Louvain-la-Neuve, Belgium, 2004.

[29] T. Calliacoudas and F. Issa, "Multiconductor transmission lines and cables solver,' An efficient simulation tool for PLC channel networks development," in Proceedings of the IEEE International Symposium on Power Line Communications and Its Applications (ISPLC '02), Athens, Greece, March 2002.

[30] S. Galli and T. Banwell, "A deterministic frequency-domain model for the indoor power line transfer function," IEEE Journal on Selected Areas in Communications, vol. 24, no. 7, pp. 1304-1316, 2006.

[31] S. Galli and T. Banwell, "A novel approach to the modeling of the indoor power line channel-part II: transfer function and its properties," IEEE Transactions on Power Delivery, vol. 20, no. 3, pp. 1869-1878, 2005.

[32] C. R. Paul, Analysis of Multiconductor Transmission Lines, John Wiley \& Sons, New York, NY, USA, 1994.

[33] J. A. B. Faria, Multiconductor Transmission-Line Structures: Modal Analysis Techniques, John Wiley \& Sons, New York, NY, USA, 1994.

[34] T. Sartenaer and P. Delogne, "Deterministic modeling of the (shielded) outdoor power line channel based on the multiconductor transmission line equations," IEEE Journal on Selected Areas in Communications, vol. 24, no. 7, pp. 12771291, 2006.

[35] T. Sartenaer and P. Delogne, "Powerline cables modelling for broadband communications," in Proceedings of the IEEE International Symposium on Power Line Communications and Its Applications (ISPLC '01), pp. 331-337, Malmö, Sweden, April 2001.

[36] A. Pérez, A. M. Sánchez, J. R. Regué et al., "Circuital and modal characterization of the power-line network in the PLC band," IEEE Transactions on Power Delivery, vol. 24, no. 3, pp. 11821189, 2009.

[37] H. Meng, S. Chen, Y. L. Guan et al., "Modeling of transfer characteristics for the broadband power line communication channel," IEEE Transactions on Power Delivery, vol. 19, no. 3, pp. 1057-1064, 2004.

[38] P. Amirshahi, Broadband access and home networking through powerline networks, Ph.D. dissertation, Pennsylvania State University, University Park, Pa, USA, 2006, http://etda. 
libraries.psu.edu/theses/approved/WorldWideIndex/ETD-1205/index.html.

[39] S. Galli, "A novel approach to the statistical modeling of wireline channels," IEEE Transactions on Communications, vol. 59, no. 5, pp. 1332-1345, 2011.

[40] S. Galli, "A simplified model for the indoor power line channel," in Proceedings of the IEEE International Symposium on Power Line Communications and Its Applications (ISPLC '09), pp. 13-19, Dresden, Germany, March 2009.

[41] S. Galli, "A simple two-tap statistical model for the power line channel," in Proceedings of the 14th IEEE International Symposium on Power Line Communications and Its Applications (ISPLC '10), pp. 242-248, Rio de Janeiro, Brazil, March 2010.

[42] F. Versolatto and A. M. Tonello, "Analysis of the PLC channel statistics using a bottom-up random simulator," in Proceedings of the 14th IEEE International Symposium on Power Line Communications and Its Applications (ISPLC '10), pp. 236241, Rio de Janeiro, Brazil, March 2010.

[43] A. M. Tonello, F. Versolatto, and C. Tornelli, "Analysis of impulsive UWB modulation on a real MV test network," in Proceedings of the IEEE International Symposium on Power Line Communications and Its Applications (ISPLC '11), pp. 18-23, Udine, Italy, April 2011.

[44] M. Antoniali, A. M. Tonello, M. Lenardon, and A. Qualizza, "Measurements and analysis of PLC channels in a cruise ship," in Proceedings of the IEEE International Symposium on Power Line Communications and Its Applications (ISPLC '11), pp. 102-107, Udine, Italy, April 2011.

[45] A. M. Tonello and F. Versolatto, "Bottom-up statistical PLC channel modeling - part II: inferring the statistics," IEEE Transactions on Power Delivery, vol. 25, no. 4, pp. 2356-2363, 2010.

[46] F. J. Cañete, J. A. Cortés, L. Díez, and J. T. Entrambasaguas, "A channel model proposal for indoor power line communications," IEEE Communications Magazine, vol. 49, no. 12, pp. 166-174, 2011.

[47] M. Tlich, A. Zeddam, F. Moulin, and F. Gauthier, "Indoor power-line communications channel characterization up to $100 \mathrm{MHz}$ - part II: time-frequency analysis," IEEE Transactions on Power Delivery, vol. 23, no. 3, pp. 1402-1409, 2008.

[48] M. Tlich, G. Avril, and A. Zeddam, "Coherence bandwidth and its relationship with the rms delay spread for PLC channels using measurements up to $100 \mathrm{MHz}$," in Proceedings of the International Federation for Information Processing (IFIP '08), pp. 129-142, December 2008.

[49] N. Suljanović, A. Mujčić, M. Zajc, and J. F. Tasič, "Highfrequency characteristics of high-voltage power line," in Proceedings of the IEEE International Conference on Computer as a Tool, pp. 310-314, Ljubljana, Slovenia, September 2003.

[50] W. Villiers, J. H. Cloete, and R. Herman, "The feasibility of ampacity control on HV transmission lines using the PLC system," in Proceedings of the 6th IEEE Africon Conference in Africa (AFRICON '02), vol. 2, pp. 865-870, George, South Africa, October 2002.

[51] M. Zajc, N. Suljanović, A. Mujčić, and J. F. Tasič, "Frequency characteristics measurement of overhead high-voltage powerline in low radio-frequency range," IEEE Transactions on Power Delivery, vol. 22, no. 4, pp. 2142-2149, 2007.

[52] J. Kuffel, E. Kuffel, and W. S. Zaengl, High-Voltage Engineering Fundamentals, Butterworth Heinemann, Woburn, Mass, USA, 2001.

[53] M. Z. A. A. Kadir, J. Sardi, W. F. W. Ahmad, H. Hizam, and J. Jasni, "Evaluation of a $132 \mathrm{kV}$ transmission line performance via transient modelling approach," European Journal of Scientific Research, vol. 29, no. 4, pp. 533-539, 2009.

[54] R. K. Z. Sahbudin, S. A. Fauzi, S. Hitam, and M. Mokhtar, "Investigation of electrical potential and electromagnetic field for overhead high voltage power lines in Malaysia," Journal of Applied Sciences, vol. 10, no. 22, pp. 2862-2868, 2010.

[55] M. D'Amore and M. S. Sarto, "A new formulation of lossy ground return parameters for transient analysis of multiconductor dissipative lines," IEEE Transactions on Power Delivery, vol. 12, no. 1, pp. 303-309, 1997.

[56] P. Amirshahi and M. Kavehrad, "Medium voltage overhead powerline broadband communications; transmission capacity and electromagnetic interference," in Proceedings of the IEEE International Symposium on Power Line Communications and Its Applications (ISPLC '05), pp. 2-6, Vancouver, Canada, April 2005.

[57] M. D'Amore and M. S. Sarto, "Simulation models of a dissipative transmission line above a lossy ground for a widefrequency range-part I: single conductor configuration," IEEE Transactions on Electromagnetic Compatibility, vol. 38, no. 2, pp. 127-138, 1996.

[58] M. D'Amore and M. S. Sarto, "Simulation models of a dissipative transmission line above a lossy ground for a widefrequency range-Part II: multiconductor configuration," IEEE Transactions on Electromagnetic Compatibility, vol. 38, no. 2, pp. 139-149, 1996.

[59] J. Anatory and N. Theethayi, "On the efficacy of using ground return in the broadband power-line communications-A transmission-line analysis," IEEE Transactions on Power Delivery, vol. 23, no. 1, pp. 132-139, 2008.

[60] J. R. Carson, "Wave propagation in overhead wires with ground return," Bell System Technical Journal, vol. 5, pp. 539554, 1926.

[61] H. Kikuchi, "Wave propagation along an infinite wire above ground at high frequencies," Electrotechnical Journal of Japan, vol. 2, pp. 73-78, 1956.

[62] H. Kikuchi, "On the transition form a ground return circuit to a surface waveguide," in Proceedings of the International Congress on Ultra High Frequency Circuits and Antenna, pp. 39-45, Paris, France, October 1957.

[63] F. Issa, D. Chaffanjon, E. P. de la Bâthie, and A. Pacaud, "An efficient tool for modal analysis transmission lines for PLC networks development," in Proceedings of the IEEE International Symposium on Power Line Communications and Its Applications (ISPLC '02), Athens, Greece, March 2002.

[64] W. Villiers, J. H. Cloete, L. M. Wedepohl, and A. Burger, "Realtime sag monitoring system for high-voltage overhead transmission lines based on power-line carrier signal behavior," IEEE Transactions on Power Delivery, vol. 23, no. 1, pp. 389395, 2008.

[65] B. S. Yarman and A. Fettweis, "Computer-aided double matching via parametric representation of brune functions," IEEE Transactions on Circuits and Systems, vol. 37, no. 2, pp. 212-222, 1990.

[66] R. Araneo, S. Celozzi, G. Lovat, and F. Maradei, "Multi-port impedance matching technique for power line communications," in Proceedings of the IEEE International Symposium on Power Line Communications and Its Applications (ISPLC '11), pp. 96-101, Udine, Italy, April 2011.

[67] E. Fortunato, A. Garibbo, and L. Petrolino, "An experimental system for digital power line communications over high voltage electric power lines-field trials and obtained results," in Proceedings of the IEEE International Symposium on Power 
Line Communications and Its Applications (ISPLC'03), pp. 2631, Kyoto, Japan, March 2003.

[68] J. Anatory, N. Theethayi, and R. Thottappillil, "Power-line communication channel model for interconnected networks - part II: multiconductor system," IEEE Transactions on Power Delivery, vol. 24, no. 1, pp. 124-128, 2009.

[69] FCC, "In the matter of amendment of part 15 regarding new requirements and measurement guidelines for access broadband over power line systems," FCC 04-245 Report and Order, 2008.

[70] NTIA, "Potential interference from broadband over power line (BPL) systems to federal government radio communications at $1.7-80 \mathrm{MHz}$ Phase 1 Study Vol. 1," NTIA Report 04-413, 2004.

[71] L. M. Kuhn, S. Berger, I. Hammerström, and A. Wittneben, "Power line enhanced cooperative wireless communications," IEEE Journal on Selected Areas in Communications, vol. 24, no. 7, pp. 1401-1410, 2006.

[72] J. Anatory, N. Theethayi, R. Thottappillil, M. M. Kissaka, and N. H. Mvungi, "The influence of load impedance, line length, and branches on underground cable power-line communications (PLC) systems," IEEE Transactions on Power Delivery, vol. 23, no. 1, pp. 180-187, 2008.

[73] J. Anatory, N. Theethayi, R. Thottappillil, M. M. Kissaka, and N. H. Mvungi, "The effects of load impedance, line length, and branches in typical low-voltage channels of the BPLC systems of developing countries: transmission-line analyses," IEEE Transactions on Power Delivery, vol. 24, no. 2, pp. 621629, 2009.

[74] OPERA1, "D5: pathloss as a function of frequency, distance and network topology for various LV and MV European powerline networks," IST Integrated Project No 507667, 2005.

[75] ITU-T SG15/Q4, Powerline channel data. Contribution NIPPNAI-2007-107R1, 2007.

[76] B. O'Mahony, "Field testing of high-speed power line communications in North American homes," in Proceedings of the IEEE International Symposium on Power Line Communications and Its Applications (ISPLC '06), pp. 155-159, Orlando, Fla, USA, March 2006. 

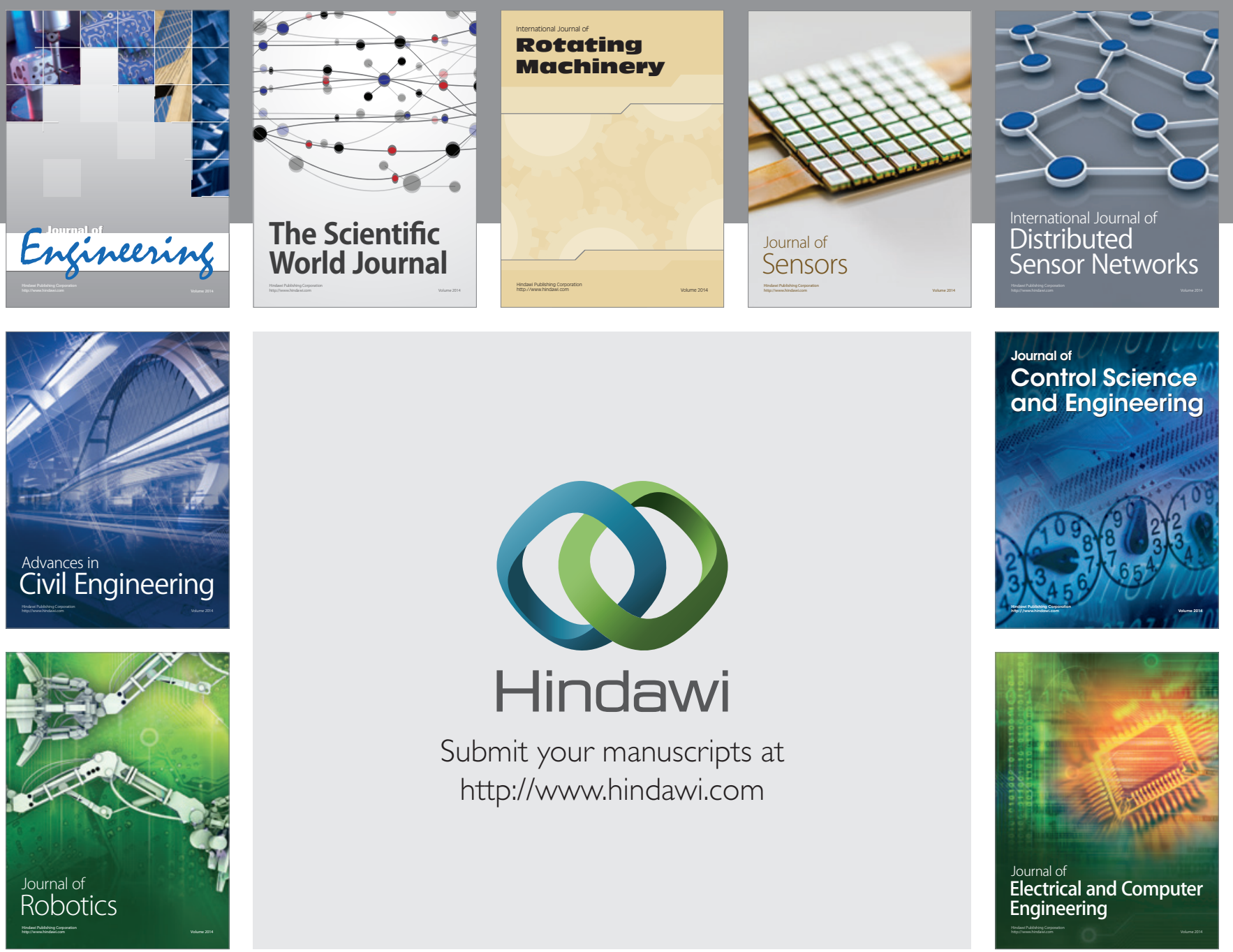

Submit your manuscripts at

http://www.hindawi.com
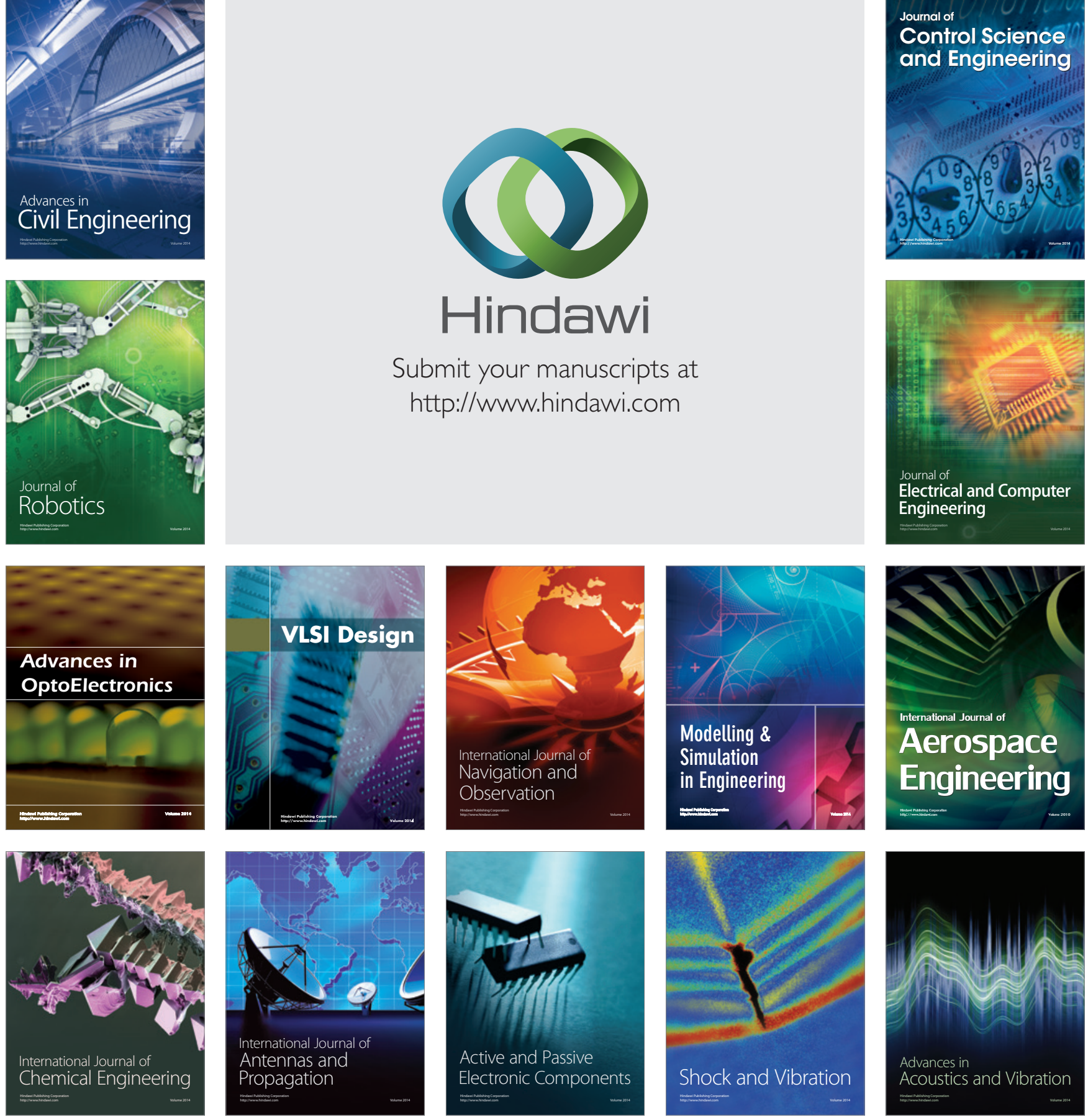